\title{
Karrierewege zur Professur an einer Fachhochschule
}

\author{
Ursula Diallo-Ruschhaupt
}

Susanne Plaumann

Eva-Maria Dombrowski

Schriftenreihe „Gender-Diskurs“ des Gender- und Technik-Zentrum (GuTZ)

der Beuth Hochschule für Technik Berlin

Band 09

Herausgeberinnen

Eva-Maria Dombrowski, Antje Ducki

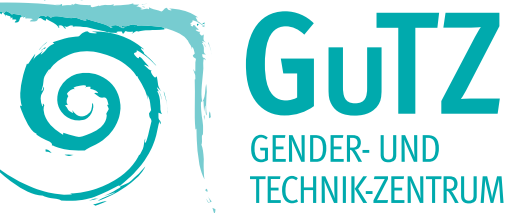




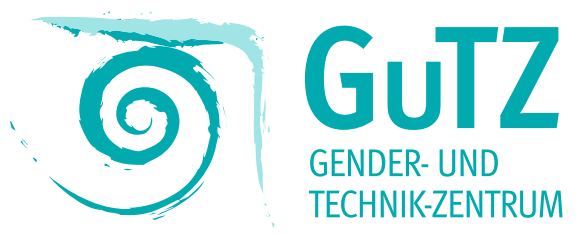

(c) 2018 Dieses Werk ist im Verlag Barbara Budrich erschienen und steht unter folgender Creative Commons Lizenz: http://creativecommons.org/licenses/by-nc-nd/3.0/de/

Verbreitung, Speicherung und Vervielfältigung erlaubt, kommerzielle Nutzung und Veränderung nur mit Genehmigung des Verlags Barbara Budrich.

\section{(cc) EY-NC-ND}

Dieses Werk steht im OpenAccess Bereich der Verlagsseite zum kostenlosen Download bereit: (https://doi.org/xyz/xyz).

Eine kostenpflichtige Druckversion (Printing on Demand) kann über den Verlag bezogen werden. Die Seitenzahlen in der Druck- und Onlineversion sind identisch.

ISBN 978-3-86388-769-8 (Druckfassung)

elSBN 978-3-86388-329-4 (eBook)

DOI: https://doi.org/10.3224/863887698

Druck: www.wirmachendruck.de

Verlag Budrich UniPress Ltd.

www.budrich-verlag.de 


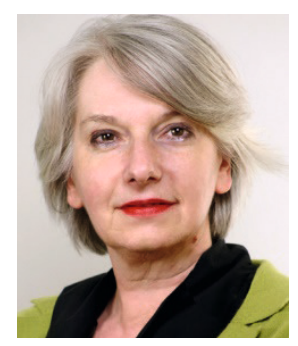

Frau Ursula Diallo-Ruschhaupt ist Diplomsoziologin mit den Schwerpunkten Wissenschafts- und Bildungssoziologie. Sie war fast 15 Jahre als wissenschaftliche Mitarbeiterin an der Beuth Hochschule für Technik im Bereich Begabtenförderung beschäftigt und maßgeblich am Aufbau des Gender-und Technik-Zentrums beteiligt. Zurzeit ist sie Mitarbeiterin bei der Gesellschaft für interkulturelles Zusammenleben Berlin.

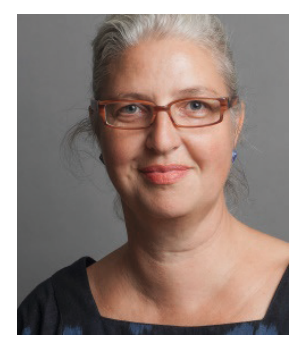

Frau Susanne Plaumann ist seit 2015 zentrale Frauenbeauftragte der Beuth Hochschule für Technik Berlin. Ausgehend von ihrer geisteswissenschaftlichen Ausbildung hat sie in Forschungsprojekten zu kulturwissenschaftlichen Fragestellungen gearbeitet. 2002 hat sie ein Doktorandinnen-Programm an der Technischen Universität Berlin konzipiert und bis 2015 verantwortet. Weitere Schwerpunkte in ihrer Gleichstellungspraxis sind Förderformate für Studentinnen in MINT-Studiengängen.

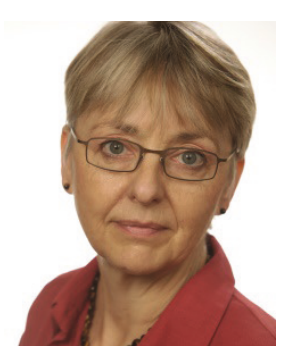

Frau Prof. Dr. Eva-Maria Dombrowski ist seit 1996 Professorin für Verfahrenstechnik an der Beuth Hochschule für Technik Berlin. Sie leitet zusammen mit Frau Prof. Dr. Antje Ducki seit 2009 das Gender- und Technik-Zentrum. Im Mittelpunkt ihrer Forschungsarbeiten stehen Umweltschutzthemen sowie Mentoring im MINT-Bereich.

\section{Gefördert durch das}

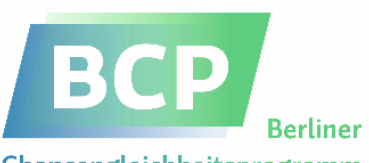

Chancengleichheitsprogramm

Diese Publikation geht auf eine Analyse- und Recherchetätigkeit zurück, die über das Berliner Chancengleichheitspro-gramm gefördert wurde.

Impressum

Herausgeberinnen:

Prof. Dr. Eva-Maria Dombrowski

Prof. Dr. Antje Ducki

Redaktion:

Gender- und Technik-Zentrum der

Beuth Hochschule für Technik

Luxemburger Str. 10

13353 Berlin

E-Mail:gutz@beuth-hochschule.de

Internet: https://www.beuth-hochschule.de/gutz/

Verantwortlich für den Inhalt sind die Autorinnen. 



\section{Vorwort - Erfolgreiche Bilanz und Kurswechsel}

Die Beuth Hochschule für Technik Berlin blickt mit ihrem Hypatia Programm und weiteren unter anderem durch das Berliner Chancengleichheitsprogramm BCP geförderten Programmteilen auf eine Tradition der erfolgreichen Frauenförderung im Bereich der wissenschaftlichen Nachwuchsförderung durch Vergabe von Promotionsstipendien an Absolventinnen der Beuth Hochschule sowie durch Besetzung von Gastdozenturen und Gastprofessuren zurück. Der bisherige Weg war einerseits geprägt davon, dem eigenen Nachwuchs den Zugang zu einer wissenschaftlichen Karriere zu ermöglichen und den Pool an aussichtsreichen Kandidatinnen unter dem speziellen Anforderungsprofil im naturwissenschaftlichtechnischen Bereich für eine Fachhochschulprofessur zu vergrößern. Auf der anderen Seite waren und sind die politischen Rahmenbedingungen und die Förderstruktur prägend, da zahlreiche Maßnahmen unter dem Aspekt Chancengleichheit der Geschlechter durch Drittmittel eingeworben werden müssen und Frauenfördermaßnahmen und Pilotprojekte eher akzeptiert werden, wenn sie aus zusätzlichen Mitteln realisiert werden können.

Die Auswertung zum Hypatia Programm der Beuth Hochschule im Teil B dieser Publikation, die sich auf 22 Jahre Förderung von Wissenschaftskarrieren herausragender MINT-Absolventinnen der Beuth Hochschule und Nachwuchswissenschaftlerinnen im MINT-Bereich bezieht, arbeitet heraus, wo die einzelnen Maßnahmen ansetzten und inwieweit und durch welche Aspekte die Karriere der geförderten Frauen positiv beeinflusst werden konnte. Auf dieser Grundlage werden Erfolgsfaktoren formuliert, die für die Weiterentwicklung der Förderstruktur der Beuth Hochschule bedeutsam sind und zum Teil in die zukünftige Frauenförderstrategie an der Beuth Hochschule einfließen.

Die folgenden Ausführungen im Teil A dieser Publikation beschäftigen sich mit den politischen Diskussionen um Karrierewege insbesondere an Hochschulen für angewandte Wissenschaften und den Empfehlungen für eine zukünftige Personalentwicklung. Daran schließt sich nach einer statistischen Auswertung der Berufungsvorgänge die kritische Auseinandersetzung mit der bisherigen Praxis der Beuth Hochschule zur Gewinnung von Professorinnen an. Auf dieser Grundlage wird die neue strategische Ausrichtung der Beuth Hochschule vorgestellt, wie sie auch im 2015 verabschiedeten Gleichstellungskonzept verankert ist und u.a. durch BCP-geförderte Projekte im Programmrahmen von 2016 bis 2020 umgesetzt werden soll.

Die Herausgeberinnen

\footnotetext{
${ }^{1}$ Hypatia Programm zur Förderung von Wissenschaftskarrieren herausragender MINT-Absolventinnen der Beuth Hochschu-le für Technik Berlin von 1994 bis 2015. Die Mittel zur Förderung des Hypatia Programms stellte der Berliner Senat, zuletzt im Rahmen des BCP, zur Verfügung (vgl. Teil B).

${ }^{2}$ Das Berliner Programm zur Förderung der Chancengleichheit für Frauen in Forschung und Lehre BCP wurde 2001 vom Berliner Senat eingerichtet und wird seitdem kontinuierlich weiterentwickelt.
} 



\section{Inhalt}

Teil A: Personalentwicklung für mehr Professorinnen an Fachhochschulen

A1 Diskussionsstand und Rahmen für die Gewinnung des wissenschaftlichen Nachwuchses 1

A1.1 Prototypische Karriereverläufe - Karriereweg Juniorprofessur 8

A1.2 Promotion von FH-Absolventinnen und FH-Absolventen $\quad 10$

A1.3 Berufungsgeschehen im Maschinenbau und der Elektrotechnik 11

A1.4 Pool an Bewerberinnen zu klein $\quad 11$

A1.5 Ansprache in der Ausschreibung entscheidend $\quad 14$

A2 Zur aktuellen Situation an der Beuth Hochschule 16

A2.1 Berufungskorridore $\quad 16$

A2.2 Proaktive Rekrutierung $\quad 18$

A2.3 Hochschule im Wettbewerb 19

A2.4 Stellenbesetzungen und Berufungen - bereits erprobte und erfolgreiche Maßnahmen $\quad 19$

A2.5 Gleichstellungskonzept der Beuth Hochschule 20

A2.6 Durchlässigkeit auch in Karrierephasen fördern $\quad 21$

A3 Gewinnung von FH-Professorinnen im MINT-Bereich 23

A3.1 Projekte im Rahmen des BCP 23

$\begin{array}{ll}\text { A3.2 Personalentwicklung als Zukunftsfeld } & 24\end{array}$

Teil B: Auswertung und Fazit zum Hypatia Programm der Beuth Hochschule -

22 Jahre Förderung von Wissenschaftskarrieren herausragender MINT-Absolventinnen der Beuth Hochschule und ingenieurwissenschaftlicher Nachwuchswissenschaftlerinnen

B1 Programmstruktur zur Nachwuchsförderung bis 2015

B1.1 Einleitung $\quad 26$

$\begin{array}{ll}\text { B1.2 Hypatia Programm } & 27\end{array}$

B1.2.1 Veränderungen des Förderkonzepts in Anpassung an Veränderungen der

B1.2.2 Förderzeitraum 2001 bis $2015 \quad 29$

B1.2.3 Personengebundene Förderung des Hypatia Programms 30

B1.2.4 Erfolge - Promotionsförderung 31

B1.2.5 Erfolge - Gastdozenturen 33

B1.2.6 Erfolge der Gastprofessorinnen $\quad 34$

B1.2.7 Zusammenstellung der Erfolge und Verbleib der Geförderten $\quad 35$

B2 Wege von Beuth Absolvierenden zur Promotion - Szenario eines Hindernislaufs 39

B3 In die Zukunft gerichtete Empfehlungen $\quad 42$

$\begin{array}{ll}\text { Literatur } & 44\end{array}$ 


\section{Personalentwicklung für mehr Professorinnen an Fachhochschulen Susanne Plaumann, Eva-Maria Dombrowski}

\section{A1 Diskussionsstand und Rahmen für die Gewinnung des wissenschaftlichen Nachwuchses}

\section{A1.1. Prototypische Karriereverlåufe - Karriereweg Juniorprofessur}

Für 2015 weist das Statistische Bundesamt an Hochschulen für angewandte Wissenschaften $(\mathrm{HAWs})^{3}$ an den Professuren einen Frauenanteil von 21,8 \% aus (StaBu 2016). Der aktuelle Stand des Frauenanteils an Professuren an Hochschulen für angewandte Wissenschaften (HAWs) ist höher als die Jahre zuvor (2000 11\%, 2005 15\%, 2010 20,1\%), allerdings fällt die Steigerungsrate im Vergleich mit den letzten Jahren geringer aus.

Für 2014 werden an Fachhochschulen ohne Verwaltungsfachhochschulen für die C3/W2-Professuren insgesamt 13.624 Positionen gelistet mit einem Frauenanteil von 20,3\%. Davon befinden sich allein in Berlin 1156 Professuren an Fachhochschulen mit einem überproportionalen Frauenanteil von 32,4\% (GWK 2015).

Das sind erfreuliche Entwicklungen, die verstärkt werden können durch die zurzeit deutschlandweit stattfindenden Diskussionen und Bemühungen zur Gewinnung des wissenschaftlichen Nachwuchses. Der „wissenschaftliche Nachwuchs“ für Professuren an einer Fachhochschule unterscheidet sich grundlegend von dem für Universitäten oder künstlerischen Hochschulen. Neben der wissenschaftlichen Eignung ist die anwendungsbezogene, berufspraktische Ausrichtung mit entsprechendem Nachweis entscheidend für eine erfolgreiche Berufung. Aufgrund fehlender Qualifikationsstellen an Fachhochschulen ist der angesprochene Nachwuchs in der Regel dort keiner vorherigen Beschäftigung nachgegangen, da an Fachhochschulen - ausgenommen für einige wenige Studiengänge an einzelnen HAWs - weder ein Promotionsrecht besteht noch ausgeprägte Forschungsprojekte flächendeckend zur Weiterqualifikation strukturell verankert sind. Befristete, sogenannte Juniorprofessuren, die jungen, hochqualifizierten Wissenschaftlern und Wissenschaftlerinnen mit Promotion den Zugang zu Forschung und Lehre an Universitäten und Hochschulen ermöglichen, ohne die übliche Habilitation, und sie damit für eine Dauerprofessur qualifizieren, werden an Fachhochschulen nicht angeboten. Auch Nachwuchsgruppenleitungen (wie z.B. das Emmy Noether-Programm der DFG) gibt es nicht. Neben diesen strukturellen Hemmnissen ist der weibliche wissenschaftliche Nachwuchs zudem durch überkommene, geschlechterstereotypische Rollen- und Kompetenzzuweisungen noch stärker von Informationsdefiziten sowie Schwierigkeiten auf diesem Karriereweg betroffen.

Die Kategorie Juniorprofessur wurde nach der Abschaffung von C1-Stellen, Qualifikationsstellen zum Erwerb der Habilitation, die sich nach der erfolgreichen Promotion anschließen ließen, als neuer Karriereweg 2002 mit der 5. HRG-Novelle ${ }^{4}$ eingeführt. Damit verbunden war auch die Hoffnung auf Erfolge zum Gleichstellungsfortschritt, mit der Ablösung der Habilitation mehr Wissenschaftlerinnen im Wissenschaftssystem zu halten und damit mittelbar den Anteil von Professorinnen steigern zu können. 2013 wurde bei den Juniorprofessuren insgesamt der anvisierte Frauenanteil von 40\% erreicht (Burkhardt et.al. 2016, S.87). Trotz zahlreicher Förderprogramme wurde die Juniorprofessur allerdings nur zögernd angenommen. Von 6000 angestrebten W1-Professuren, waren bis 2013 nur knapp 1600 realisiert worden. In den Ingenieurwissenschaften spielt sie bis heute kaum eine Rolle. Mittlerweile sind die Karriereverläufe von ehemaligen Juniorprofessuren evaluiert worden und zusammenfassend lässt sich festhalten, dass die W1-Professur geeignet ist, um eine universitäre Professur zu erreichen. Für eine Berufung auf eine Professur an einer Fachhochschule spielt sie hingegen keine Rolle ${ }^{5}$.

Der Karriereweg zur Fachhochschulprofessur über eine Promotion und einschlägige, mehr-

\footnotetext{
${ }^{3}$ Im Folgenden Fachhochschulen genannt

4 5. Gesetz zur Änderung des Hochschulrahmengesetzes (HRG), in Kraft getreten 2002 (BGBI. I S. 693)

${ }^{5}$ Rund $70 \%$ der Juniorprofessorinnen und -professoren bewerben sich in der Laufzeit der Stelle auf eine universitäre Professur. W1-Professuren im MINT-Bereich sind signifikant häufiger mit einem Tenure Track ausgestattet (Burkhardt et.al. 2016, S. 94-102).
} 
jährige Berufserfahrung ist im MINT-Bereich gängige Praxis - dies gilt auch für universitäre Professuren - und geht einher mit einer hohen Reputation und sehr guten Positionen und Verdienstmöglichkeiten in Wirtschaft und Industrie. Die Auslösung aus diesen hochrangigen Industriepositionen hin auf eine nach W2 dotierte FH-Professur bereitet zudem Schwierigkeiten, kann aber auch als Chance genutzt werden, wenn ein Standbein in der Berufspraxis beibehalten wird. Berufliche Handlungsspielräume werden dann höher eingeschätzt, als wenn eine Fixierung auf das Berufsfeld Wissenschaft erfolgt.

Die Fachhochschule ist aber weiterhin als „place to be“ in Berufsorientierungsprozessen einerseits wenig bekannt und andererseits müssen hier zur Rekrutierung, anders als an Universitäten, die über Promotions- bzw. Post-Doc-Stellen möglichen professoralen Nachwuchs generieren, insbesondere Interessentinnen für das Berufungsverfahren auf eine Professur tatsächlich „neu“ von außen angeworben werden. Daher haben sich personalisierte Förderinstrumente wie Promotionsförderung, Gastdozenturen und Gastprofessuren als zielführend erwiesen, um $\mathrm{FH}$-Absolventinnen letztendlich für eine $\mathrm{FH}$-Professur zu gewinnen. Nicht zu unterschätzen ist dabei die Tatsache, dass diese unter anderem auch durch externe Mittel eingeworbenen, befristeten Positionen geeignet waren, die Hochschule in der Umsetzung ihrer Fächerkultur bis hin zur akademischen Selbstverwaltung praktisch kennen zu lernen ${ }^{6}$.

Die Gewinnung von FH-Professorinnen und Professoren wird seit der zweiten Jahreshälfte 2016 von Wissenschaftsorganisationen wie dem Wissenschaftsrat breit diskutiert und es wird bundesweit nach neuen Ideen und Ansätzen gesucht, das sogenannte Nachwuchsproblem zu lösen (Wissenschaftsrat 2016). Die Konferenz der Frauen- und Gleichstellungsbeauftragten bringt die Risikohaftigkeit dieses Karriereweges prägnant auf den Punkt:

„Karrierewege an Fachhochschulen (FHs) bzw. Hochschulen für angewandte Wissenschaften (HAWs) unterscheiden sich grundlegend von denen an Universitäten und künstlerischen Hochschulen. Die besondere Herausforderung der Qualifikation für eine Fachhochschulprofessur liegt in der spezifischen Verbindung einer wissenschaftlichen mit einer berufspraktischen oder anwendungsorientierten Eignung ohne dass an den Fachhochschulen ausgewiesene Qualifikationsstellen zur Verfügung stehen. Entsprechend ist dieser Karriereweg noch stärker als an Universitäten durch Brüche und Intransparenz sowie fehlende Planungssicherheit gekennzeichnet.“ (BUKOF 2016)

Die folgende Graphik zeigt, wie im Idealfall Karrierewege verlaufen können.

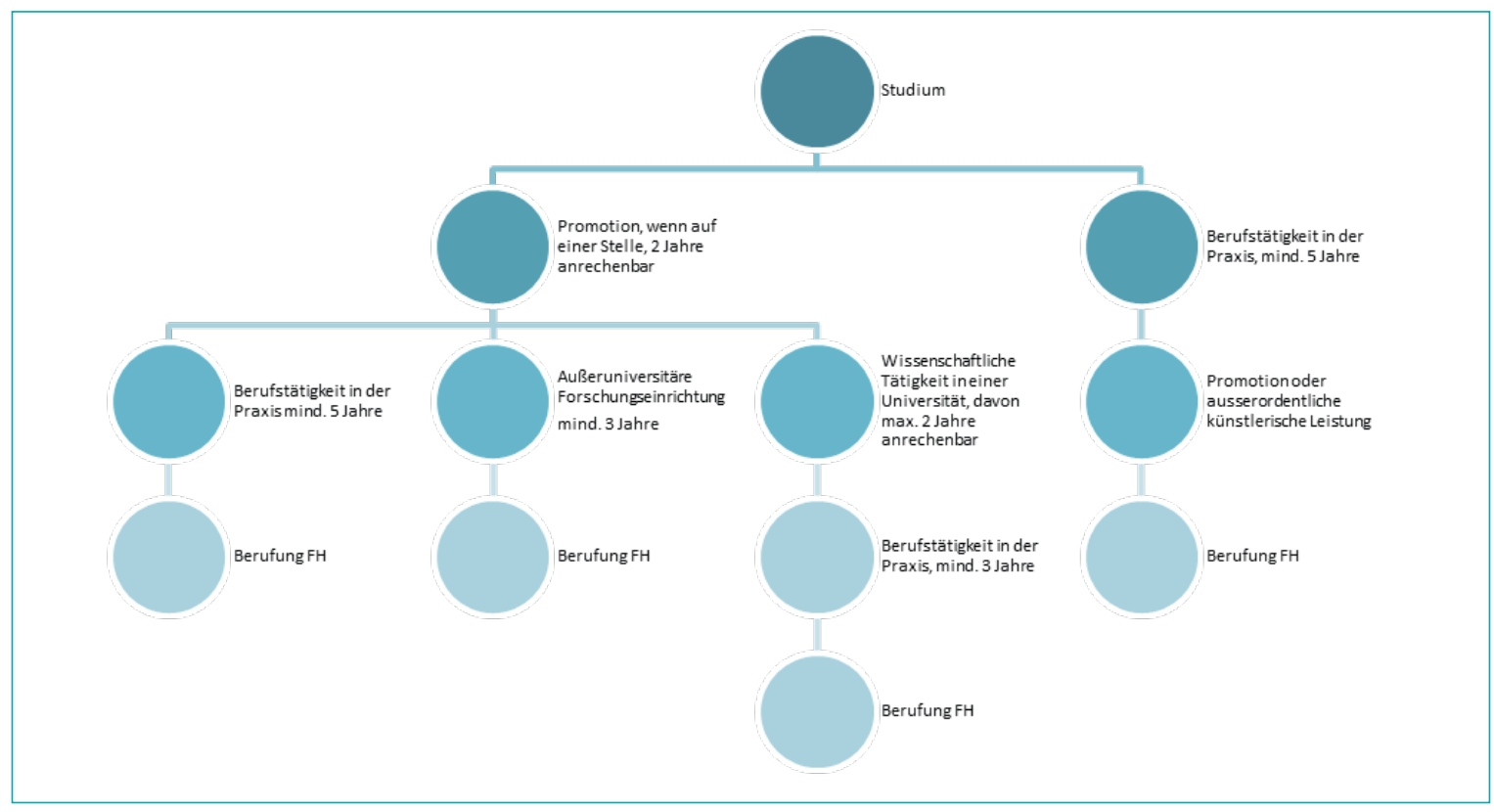

Abbildung 1: Karrierewege bis zur Berufung

FH-Absolventinnen gehen in der Mehrheit wie ihre männlichen Kommilitonen im Anschluss an

${ }^{6}$ Auswertung und Fazit zum Hypatia Programm der Beuth Hochschule - Ursula Diallo-Ruschhaupt, Teil B, S. 23 ff 
den Hochschulabschluss direkt in die berufliche Praxis. Die Zulassung zur Promotion mit FHAbschluss vor dem Bologna-Prozess war in den Promotionsordnungen der Universitäten bzw. Hochschule geregelt und häufig beschwerlich und nur mit hohem Aufwand für Zusatznachweise möglich, Wege zur Promotion für FH-Absolventinnen und FH-Absolventen waren daher kaum bekannt ${ }^{7}$. Daten zur Promotionsabsicht und zum Promotionsverlauf für FH-Absolventinnen und FH-Absolventen wurden bisher auch an der Beuth Hochschule nicht systematisch erhoben. Aus der Auswertung des Hypatia-Programms liegen erste Hinweise zu Erfolgsfaktoren im Promotionsverlauf vor. Politisch wird angestrebt den Anteil von kooperativen Promotionen zu erhöhen, ablesbar beispielsweise für Berlin an den Berliner Hochschulverträgen bis 2017, wo dies als Leistungsparameter verankert ist.

Mit universitärem Abschluss sind die Wege zur Promotion bereits etabliert und auf Stipendienbasis, auf einer Qualifikationsstelle, parallel zu einem Drittmittelprojekt oder einer Berufspraxisphase stärker im Karrieredenken der Absolventinnen und Absolventen präsent. Aus der Berufspraxis eine Promotion anzuschließen, ist möglich, aber die berufsbegleitende Promotion ist seltener. Faktisch liegen allerdings keine detaillierten Analysen zu den unterschiedlichen Karriereverläufen vor.

\section{A1.2 Promotion von FH-Absolventinnen und FH-Absolventen}

Die Umfrage der Hochschulrektorenkonferenz (HRK) zu Promotionen von FH-Absolventinnen und FH-Absolventen liefert wichtige erste Ergebnisse zu der zahlenmäßigen Verteilung und den aktuellen Entwicklungsperspektiven (HRK 2013). Die absolute Zahl von erfolgreich abgeschlossenen Promotionen von FH-Absolventinnen und FH-Absolventen steigt insgesamt, ihr Anteil hingegen an der Gesamtzahl der Promotionen beträgt weiterhin ca. 1 Prozent. Die größte Fachgruppe ist bei Zulassungen und erfolgreich abgeschlossenen Verfahren in den Ingenieurwissenschaften zu finden. Der Frauenanteil bei den abgeschlossenen Promotionen in den Ingenieurwissenschaften beträgt $17 \%$ und liegt damit allerdings erheblich unter dem Anteil an den gesamten Promotionen von 38\%.

Betrachten wir beispielhaft die erfolgreich abgeschlossenen Promotionen an der Technischen Universität Berlin, so wird sehr deutlich, wie klein die Fallzahlen tatsächlich sind: Liegt der Frauenanteil bei den Promotionen mit universitären Abschluss über sechs Jahre betrachtet durchschnittlich bei 30\%, so ist der Anteil von Promotionen von Frauen bei Fachhochulabsolvierenden insgesamt bei $26 \%$. Die Technische Universität Berlin ist ein geeigneter Bezugsrahmen, da sie einen erheblichen Pool an potentiellen geeigneten Kandidatinnen und Kandidaten für eine Professur an der Beuth Hochschule bereitstellt, wie zurückliegende Berufungsverfahren zeigen, ohne dass dies systematisch erhoben wurde.

\begin{tabular}{|c|c|c|c|c|}
\hline \multicolumn{5}{|c|}{ Promotionen an der Technischen Universität Berlin ${ }^{8}$} \\
\hline \multirow[t]{2}{*}{ Jahr } & \multicolumn{2}{|c|}{ Universitärer Abschluss } & \multicolumn{2}{|c|}{ Fachhochschulabschluss } \\
\hline & Gesamt & $\begin{array}{r}\text { Anteil-W } \\
\%\end{array}$ & Gesamt & Anteil-W \% \\
\hline 2009 & 363 & 26,4 & 13 & 38,5 \\
\hline 2010 & 455 & 29,2 & 15 & 20,0 \\
\hline 2011 & 459 & 32,9 & 21 & 23,8 \\
\hline 2012 & 443 & 29,6 & 10 & 44,4 \\
\hline 2013 & 457 & 32,6 & 19 & 21,1 \\
\hline 2014 & 497 & 30,4 & 20 & 15,0 \\
\hline Summe & 2674 & 30,3 & 106 & 26,4 \\
\hline
\end{tabular}

Tabelle 1: Promotionen nach universitärem und Fachhochschulabschluss

\footnotetext{
${ }^{7}$ Ein Leitfaden zur Promotion wurde innerhalb des Hypatia Programms an der damaligen Technischen Fachhochschule TFH erarbeitet, vgl. Teil B.

${ }^{8}$ Diese Daten werden über ein TU-internes Programm regelmäßig bis hinunter auf die Fachgebietsebene erhoben
} 
An der Beuth Hochschule erfolgt die Gewinnung von Bewerberinnen und Bewerbern für eine Professur nach $\S 100$ BerlHG u.a. mit ausreichender Praxiserfahrung. Dies ist insbesondere für den MINT-Bereich eine komplexe Herausforderung, da nur die „Doppelqualifikation“, nämlich auf wissenschaftlichem und berufsorientierten Gebiet, eine Bewerbung aussichtsreich werden lässt.

Einige Fachhochschulen haben auf den Mangel an geeigneten qualifizierten Bewerberinnen und Bewerbern bereits mit eigenen Personalentwicklungskonzepten und neuen Förderformaten reagiert: z.B. mit sog. FH-Nachwuchsprofessuren - halb in der Lehre und halb im Unternehmen verankert - an der Fachhochschule Münster, oder die FH-Nachwuchsprofessur an der Hochschule Ostwestfalen-Lippe.

Die Nachwuchsprofessur bietet die Möglichkeit, die erforderlichen drei Jahre Praxiserfahrung außerhalb einer Hoch $\neg$ schule nach erfolgreicher Promotion und gleichzeitig weitere Kompetenzen in Lehre und Forschung zu erwerben. Die Hochschule unterstützt die Kandidatinnen und Kandidaten bei der Suche nach einer geeigneten Stelle in der Wirtschaft. Für den weiblichen Nachwuchs gibt es darüber hinaus weitere Anreize: in einem hochschulinternen Förderprogramm stellt die Hochschule eine $75 \%$ Stelle zur Verfügung, sodass die Unternehmen nur $25 \%$ finanzieren müssen, um eine halbe Stelle mit einer hoch qualifizierten Nachwuchskraft zu erhalten. Damit die Hochschule selbst von dieser Förderung langfristig profitieren kann, besteht die Möglichkeit eine Nachwuchsprofessur von vornherein mit einem Tenure Track ${ }^{9}$ aus zu statten. In der Berufungsordnung ist verankert, dass die Nachwuchsprofessur in Ausnahmefällen eingesetzt werden kann, wenn z.B. bereits vorangegangene Berufungsverfahren gescheitert sind. Damit wird gewährleistet, dass der übliche Qualifikationsweg bestehen bleibt.

Ostwestfalen-Lippe hat seine FH-Nachwuchsprofessur in ein zweistufiges „Drei-Plus-Fünf- Modell“ gegliedert. In einer ersten Phase wird die berufspraktische Komponente als Lehrkraft für besondere Aufgaben im Rahmen einer Teilzeitbeschäftigung von 50\% nach EG 13 TV-L mit 5 SWS erworben. Qualitätssicherung erfolgt durch die Personalauswahl im Rahmen einer üblichen Berufungskommission und eines Qualifizierungsplanes. Parallel dazu wird ein Beschäftigungsverhältnis von 50\% mit einer einschlägigen hochschulexternen Einrichtung oder einem Unternehmen eingegangen. Mit dem Abschluss der dreijährigen ersten Qualifizierungsphase sind die notwendigen Voraussetzungen für eine Professur erfüllt und durch externe Gutachten sowie eine Lehrevaluation überprüft, sodass das Dienstverhältnis im zweiten Qualifikationsabschnitt zunächst auf eine W2-Professur auf Zeit überführt werden kann (HS-OWL 2017).

\section{A1.3 Berufungsgeschehen im Maschinenbau und der Elektrotechnik}

Für die technischen Fachdisziplinen lässt sich feststellen, dass die Zufriedenheit mit der Qualität und Quantität von Bewerbungen in den Ingenieurwissenschaften nicht allzu hoch ist, wie z.B. die Befragung im Februar 2015 von Dekaninnen und Dekanen in den Fachbereichen des Maschinenbaus und der Elektrotechnik zeigen (VDMA 2015, S.13). An Fachhochschulen sind diese zu 70\% unzufrieden oder sogar sehr unzufrieden mit der Quantität der Bewerbungen (VDMA 2015, S.13, Abb.3). Die Qualität der Bewerbungen wird hingegen von allen Befragten hochschultypübergreifend als überwiegend zufriedenstellend oder teil/teils zufriedenstellend eingeschätzt. Die Relevanz von Industrieerfahrungen wird für die Aufnahme in ein Auswahlverfahren insgesamt als sehr hoch bewertet, allerdings kommt Forschungsprojekten und der Einwerbung von Drittmitteln in der Wahrnehmung der Befragten in den letzten fünf Jahren mehr Bedeutung zu als früher. Bei den berufenen Professorinnen und Professoren geben 32\% der Dekaninnen und Dekane an Fachhochschulen an (im Vgl. zu 22\% an Universitäten), dass mehr als $75 \%$ Industrieerfahrungen haben. Das übrige Viertel wird vermutlich ihre Praxiserfahrungen an außeruniversitären Forschungseinrichtungen erworben haben (VDMA 2015, S.14).

\section{A1.4 Pool an Bewerberinnen zu klein}

Um potentielle Kandidatinnen bundesweit sichtbar(er) zu machen, wurden Datenbanken wie z.B. femconsult etabliert, die es erlauben, über eine Suchmaske innerhalb kürzester Zeit eine

\footnotetext{
${ }^{9}$ „Verfahren zur Einstellung“, Tenure-Track-Professur: nach erfolgreicher Bewährungsphase erfolgt unmittelbar die Überlei-tung in eine Lebenszeitprofessur, https://www.bmbf.de/de/wissenschaftlicher-nachwuchs-144.html
} 
Abfrage durchzuführen und über dieses Portal eine Liste potentieller Kandidatinnen zu generieren. Kommissionsmitglieder, Dekaninnen und Dekane und Gleichstellungs- und Frauenbeauftragte können so aktuelle Ausschreibungen von Professuren persönlich per Email an geeignete Kandidatinnen adressieren und sie zur Bewerbung motivieren ${ }^{10}$. In die Datenbanken können sich Nachwuchswissenschaftlerinnen mit ihrem Qualifikationsprofil selbstständig eintragen.

Informationen zu Berufungsvoraussetzungen und Zusammensetzung von Berufungskommission stehen im Netz vielfach zur Verfügung, wie z.B. auf den Seiten der Bundeskonferenz der Frauen- und Gleichstellungsbeauftragten an Hochschulen ${ }^{11}$, aber auch Projekte, wie der Zusammenschluss nord- und mitteldeutscher Hochschulen namens „PROfessur“. „PROfessur“ ist an der Hochschule Hannover angesiedelt und bietet auf seinen Seiten neben den Basisinformationen zur FH-Professur und Listen mit Fachvertreterinnen, die von Interessentinnen kontaktiert werden können, auch einen Verteiler mit Kontakten zu Nachwuchswissenschaftlerinnen, über die alle Ausschreibungen der Mitgliedshochschulen versendet werden ${ }^{12}$.

Dennoch besteht weiterer Handlungsbedarf, um den Pool an Bewerberinnen zu erhöhen. Der Prozessablauf für ein Berufungsverfahren an der Beuth Hochschule für Technik Berlin ist im Folgenden dargestellt:

- Stelle vakant

- Beschluss Fachbereichsrat (FBR) zur Wiederbesetzung

- Stellenzuweisung mit Kapazitätsberechnung durch Akademischen Senat (AS)

- Hochschulöffentliche und öffentliche Ausschreibung der Stelle, ggf. Wiederholung bei fehlenden weiblichen Bewerbungen

- Bildung einer Berufungskommission (BK)

- Konstituierung der BK - Arbeit der BK

- Erarbeitung des Kriterienkatalogs zur Auswahl (Matrix)

- Sichtung aller Bewerbungsunterlagen und Entscheidung über einzuladende Bewerberinnen und Bewerber

- Einladung der Ausgewählten, ein Termin ggf. zwei Termine an der Hochschule

1. Termin: Vorgespräch mit der BK

2. Termin: Probevorlesung, Fachvortrag (teilweise in engl. Sprache), Gespräch mit der BK

- Entscheidung über Listenplatzierung (Reihenfolge) und Beschlussvorschlag durch die BK

- Einholen von empfehlenden oder vergleichenden Gutachten

- Beschluss des Berufungsvorschlages durch Fachbereichsrat

- Weiterleitung der Liste an den Akademischen Senat (AS)

- Prüfung im Präsidium und durch die Kommission zur Stellungnahme zu Berufungsvorschlägen (KSB)

- Vorstellung des Vorgangs im AS durch KSB

- Empfehlung des AS mit Befürwortung an SenBWF, die sogenannte Dreierliste, oder Rückgabe an Berufungskommission

- Berufung durch die Senatorin/den Senator an die Hochschule

Ausschreibungen von Professuren werden also nicht nur in regionalen und überregionalen Zeitschriften, sondern auch in Fachjournalen publiziert, zunehmend läuft die Akquise über

\footnotetext{
${ }^{10}$ Wissenschaftlerinnen-Datenbank des Kompetenzzentrums Frauen in Wissenschaft und Forschung CEWS, http://www.gesis.org/ femconsult/recherche/

${ }^{11}$ BuKoF: Auf dem Weg zur FH-Professorin. Tipps und Informationen für Bewerberinnen. http://www.bukof.de/Handreichungen.html

12 PROfessur . Ein Projekt norddeutscher Hochschulen, http://professur.fh-hannover.de/index1.html
} 
Datenbanken und Emailverteiler oder sogar über soziale Netzwerke wie Xing ${ }^{13}$. Die Ausschreibungsorgane scheinen nicht das Problem zu sein, es fehlen dagegen Informationen, die über informelle Netzwerke bewegt werden. Diese Schlussfolgerung lässt sich aus Gesprächen mit Interessentinnen für eine Professur ziehen, aber auch aus Gesprächen, die ein sog. Headhunter (Grote, 2016) mit potentiellen Bewerberinnen geführt hat und der Arbeitsgruppe der FH-Frauenbeauftragten im Rahmen der Jahrestagung 2016 in Brandenburg vorgestellt hat.

Die Personalberaterin Frau Dr. Grote hat in ihrer Akquise Tätigkeit von den angesprochenen weiblichen Nachwuchskräften erfahren (Grote, 2016, S. 2ff), dass sie die Ausschreibungen selbst in den Printmedien und Onlineportalen nicht gesehen hatten. D. h., mit den von ihnen ausgewählten Ausschreibungsorganen wurde von Hochschulen eine potentielle Zielgruppe nicht erreicht. Nur sehr kleine Fachgruppen gewährleisten eine Sichtbarkeit und eine potentielle Kandidatin muss selbst aktiv suchen. Die in den meisten Ausschreibungen enthaltenen Passagen, dass die Bewerbung von Frauen erwünscht sei, wird von den Wissenschaftlerinnen als unverbindlicher Allgemeinplatz angesehen und wirkt daher nicht motivierend oder aktivierend. Die familiäre Belastung ist häufig genanntes Hindernis, weshalb potentielle Kandidatinnen von einer Bewerbung absehen, die neben der beruflichen Verpflichtung die Hauptlast der Familienverantwortung tragen. Dies erschwert insbesondere Hochschulen jenseits der Metropolen eine erfolgreiche Akquise. Das Gehaltsgefälle zwischen derzeitiger Beschäftigungsposition und Professur ist relevant, für Frauen mit familiären Teil- und Auszeiten aber weniger bedeutsam. Frauen sind überwiegend nur dann zu einer Bewerbung bereit, wenn sie der Überzeugung sind, den Stellenanforderungen in großem Umfang zu entsprechen. Aufgrund einer geringen Bewerberinnen- und Bewerberzahl beschließen Berufungskommissionen oftmals, das Anforderungsprofil im laufenden Verfahren abzuschwächen. Potentielle Bewerberinnen, die die in der Ausschreibung formulierten Anforderungen als zwingend einstufen, sind in solchen Fällen dann gar nicht erst im Bewerbungspool. Ein Teil der angesprochenen Frauen, die bereits Erfahrungen mit Berufungsverfahren hatten, waren der Überzeugung, lediglich zu Alibizwecken zu den Vorträgen eingeladen worden zu sein.

Frau Dr. Grotes Empfehlungen münden darin, Berufungsverfahren besser vorzubereiten und vor allem die Ausschreibungsfristen nicht in Urlaubs- und Ferienzeiten zu legen, wo die Familienbelastung besonders hoch ist. Das Nachfolgemanagement sollte wesentlich früher beginnen und zum Beispiel „Findungssymposien“ vorschalten, um das Bewerberinnenfeld auf diese Weise mittelfristig zu sondieren. Auch der Umgang mit nicht berücksichtigten Bewerberinnen sollte Bestandteil eines umfassenden Personalmarketings sein und insbesondere Feedbackgespräche vorsehen, um die Motivation qualifizierte Wissenschaftlerinnen an einer Professur zu halten oder gar zu verstärken.

Die Akquise von potentiellen Bewerberinnen gelingt nicht ausschließlich über diese etablierten Wege, sondern es sind aktivere Formen notwendig. Wenn die Hochschulen nicht über Berufungsbeauftragte mit eigener Stelle und Ressourcen verfügen oder die Berufungskommission erst nach Ende der Ausschreibungsfrist gebildet wird und die Kommissionsmitglieder aufgrund von Lehr- und Verwaltungstätigkeiten weder Zeit für Identifikation und aktive Ansprache von potentiellen Bewerberinnen noch Kenntnis vom Personalmarketing haben, entsteht ein Problem mit der Ausgestaltung des Berufungsprozesses, da niemand für die aktive Rekrutierung verantwortlich ist oder diese erst zu spät einsetzt.

Geeignete Kandidatinnen müssen persönlich angesprochen werden, denn erst im Verlauf von Gesprächen reift der Entschluss, sich zu bewerben. „Dabei ist das Potential an qualifizierten Frauen, selbst in den Ingenieurwissenschaften, durchaus vorhanden. Könnte man dieses Potential heben und mehr qualifizierte Frauen zu einer Bewerbung auf eine vakante Professur ermutigen, würde dies die Auswahlmöglichkeiten von Berufungsgremien und damit die Qualität der Hochschulausbildung erhöhen.“ (Grote, 2016, S. 1). Dies entspricht hingegen häufig nicht dem zeitlichen Aufwand, der betrieben werden möchte oder gar der Vorstellung der Mitglieder der Berufungskommission insbesondere der Statusgruppe der Professorinnen und Professoren: Sie wollen Bewerbungen von Menschen, die es wirklich wollen, also stark intrinsisch motiviert sind. Dabei wird ausgeblendet, dass Karrieremuster und Motivation von männlichen

${ }^{13}$ Xing, https://www.xing.com/ 
Nachwuchswissenschaftlern vergleichsweise stark in der Erwartungshaltung präsent und über Ausschreibungsanforderungen abgebildet sind, sodass diese häufig zu diesem Zeitpunkt der Karriere (Selbst)Zweifel an einer Passgenauigkeit gar nicht erst zu kennen scheinen. Diese Haltung von potentiellen Berufungskommissionsmitgliedern, auch wenn sie zunächst passiv erscheint, unterstützt daher den aktiven Ausschluss von Nachwuchswissenschaftlerinnen. Sehr gut wurde dies von der Soziologin Sandra Beaufaÿs in ihrer Dissertation herausgearbeitet.

„Wissenschaft ist eine soziale Angelegenheit, d.h. sie funktioniert nur über die Gemeinschaft derer, die sich gegenseitig als vollwertige Mitglieder derselben anerkennen; bereits anerkannte Wissenschaftler führen andere in die Gemeinschaft ein; wissenschaftliche Arbeit und ihre Qualität wird durch die Akteure verkörpert; Qualitätsurteile sind gebunden an übereinstimmende Sichtweisen; Leistungen werden Personen zugeschrieben. Da Leistungen und somit die Qualität wissenschaftlicher Arbeit im Anerkennungssystem der Wissenschaft immer auch verkörpert werden durch die Akteure, muss es neu Hinzukommenden gelingen, als Akteure wahrgenommen zu werden, indem die anderen ihnen solche Leistungen zuschreiben. Und hier sind Frauen in größeren und anderen Schwierigkeiten als ihre männlichen Kollegen.“ (Beaufaÿs 2004, Abs. 10)

„Wie ich einführend angedeutet habe, verstehe ich den Prozess des Wissenschaftlerwerdens vor allem als alltäglich sich vollziehende praktische Auseinandersetzung sowohl mit Forschungsgegenständen und -material als auch mit Kollegen und Kolleginnen sowie mit den sozialen Organisationsstrukturen, Prüfungsverfahren an Universitäten und dem Anerkennungssystem einer jeweiligen scientific community. Am Ende einer solchen Auseinandersetzung stehen konkrete Akteure, die den Glauben des Feldes auf spezifische Weise verkörpern. Mit diesem spezifischen Selbstverständnis wiederum bestimmen sie darüber mit, was im Feld wichtig sein soll und worum gespielt wird. Und damit werden sie schließlich zu gatekeepern für diejenigen, die neu hinzukommen.“ (Beaufaÿs 2004, Abs. 14)

Daher wird die persönliche Ansprache von qualifizierten Nachwuchswissenschaftlerinnen durch Professorinnen und Professoren für das probate Mittel gehalten, bereits im Vorfeld einer Ausschreibung zu signalisieren, dass eine Nachwuchswissenschaftlerin als „Kollegin“ und damit Bewerberin durch professorale Mitglieder akzeptiert werde. Dies kann eine Frauenbeauftragte nicht mit gleicher Wirkung leisten, auch wenn sie Interessentinnen in ihrer Reflexion „Ist diese Professur für mich interessant“ nachhaltig unterstützen kann. Anrufe von Interessentinnen, die sich z.B. danach erkundigen, ob es tatsächlich eine „offene“ und damit faire Ausschreibung ist, sind nicht selten.

\section{A1.5 Ansprache in der Ausschreibung entscheidend}

Dass die Form der Ansprache auf Männer und Frauen unterschiedlich wirkt, wurde aus verschiedenen Forschungsergebnissen im Projekt der Technischen Universität München in der Broschüre praxisorientiert und pointiert herausgearbeitet. Der gleichberechtigte Einbezug beider Geschlechter in der Stellenausschreibung wird von Männern und Frauen positiv wahrgenommen. Aber: Frauen sind weniger motiviert, sich auf „typisch männlich“ formulierte Stellenausschreibungen zu bewerben (AuBeFuehr). Formulierungen wie „durchsetzungsstark“ sind weniger ansprechend als z.B. „Teamorientierung“.

Im Handbuch „Personalauswahl in der Wissenschaft. Evidenzbasierte Methoden und Impulse für die Praxis“ werden in differenzierterer Form Forschungsergebnisse zu Geschlechtereffekten analysiert und vorgestellt und daraus Empfehlungen abgeleitet. „Die Stellenanzeige ist vielleicht das wichtigste Rekrutierungsinstrument. Sowohl der Inhalt als auch die Gestaltung haben einen starken Einfluss darauf, wie attraktiv Sie als Wissenschaftsorganisation, Forschungsteam oder Abteilung wahrgenommen werden und wer sich auf die Position bewirbt. Über eine Stellenausschreibung kann vieles gesteuert werden.“ (Peus et.al. 2015, S. 79)

Aus Sicht der Hochschule wird häufig unterschätzt, wie wenig Interessierte über die Hochschule und die ausgeschriebene Position wissen. Daher kann die Stellenausschreibung zu einer grundlegenden Quelle werden, um etwas über die Organisation und Umfeld zu erfahren. Stellenanzeigen sollten sich grundlegend am Anforderungsprofil orientieren und spezifische Aussagen dazu treffen, welche Eigenschaften oder Fähigkeiten Bewerbende mitbringen sollten 
und diese mit Hilfe von Beispielen erläutern. Studien belegen, dass Stellenanzeigen die wahrgenommene Attraktivität einer Organisation, die Glaubwürdigkeit der dargestellten Informationen und damit die Bewerbungsabsicht beeinflussen (Peus et.al. 2015, S. 72). Je nach Fachgruppe sind bei der graphischen Gestaltung der Stellenanzeigen unterschiedliche Merkmale zu beachten: für Menschen aus dem MINT-Bereich müssen Informationen zügig erfassbar sein, zentrale Punkte sind herauszuheben und lange Fließtexte sind zu vermeiden.

Studien belegen, dass Frauen und Männer Stellenanzeigen unterschiedlich lesen und vor allem bewerten. Für Männer ist das Unternehmensprofil bedeutet, Frauen interessieren sich stärker für Arbeitszeiten und Weiterqualifikationsmöglichkeiten. Im Vergleich der Passung zu einem Anforderungsprofil bewerben sich Frauen erst mit einer sehr hoch wahrgenommenen Übereinstimmung zwischen den persönlichen Kompetenzen und Eigenschaften und den Stellenanforderungen. Wenn die Organisation Diversität anstrebt, sollte dies glaubwürdig und ernsthaft Berücksichtigung erfahren, um Minoritäten zu einer Bewerbung zu ermutigen. Informationen zu Kultur und Werten der Wissenschaftsorganisation in der Stellenanzeige können die Attraktivität beeinflussen. Das Darstellen einer kooperativen Arbeitshaltung wird andere Menschen ansprechen, als die Betonung einer konkurrenzbetonten Arbeitshaltung im Kollegium (Peus et.al. 2015, S. 78ff).

Das Verfassen eines Ausschreibungstextes, der ein breites Feld an möglichen Bewerberinnen und Bewerbern anspricht, ist eine hohe Kunst, zumal der Abstimmungsprozess innerhalb der akademischen Gremien differenzierten Interessen folgt. Der Ausschreibungstext muss also einen Konsens ermöglichen, sollte das Aufgabenprofil präzise fassen, nach Möglichkeit die Hochschule charakterisieren, die Entwicklungsmöglichkeiten aufzeigen und unterschiedliche mögliche Bewerberinnen und Bewerber gleichermaßen ansprechen. Gelingt das in Fachdisziplinen einfacher, die sich der Profession nach mit Managementprozessen auseinandersetzen, so ist das in Fachkulturen mit explizit technisch-praktischem Anwendungsbezug schwieriger. Zudem sehen nicht alle Hochschullehrende eine große Anzahl an Bewerbungen als Vorteil an, da es auch eine größere Anzahl an weniger qualifizierten Bewerbungen mit sich bringen könnte, was einen höheren Aufwand bedeuten könnte. ${ }^{14}$

${ }^{14} \mathrm{vgl.} \mathrm{Jeschke/lhsen} \mathrm{S.} 58$ 


\section{A2 Zur aktuellen Situation an der Beuth Hochschule}

\section{A2.1 Berufungskorridore}

Die Beuth Hochschule weist mit dem Frauenanteil von 19\% bei den Professuren für eine Hochschule mit technischem oder „MINT“-Profil eine typische Verteilung auf. Im September 2016 standen 57 Professorinnen 240 Professoren gegenüber, die Verteilung über die Fachbereiche weist eine Streuungsbreite des Frauenanteils unter den Professuren von 5\% bis zu 31\% auf (vgl. Tabelle 2).

In den größten Fachbereichen sind dabei auch meist die Fachgebiete vertreten, die eine deutliche Überrepräsentanz von Professoren aufweisen, das sind im Detail folgende Fachbereiche:

Fachbereich III: Bauingenieur- und Geoinformationswesen mit den Bachelor- und Master-Studiengängen Bauingenieurwesen, Geoinformation, Kartographie und Geomedien, Umweltingenieurwesen Bau, Vermessungswesen und Geomatik bei einem Anteil von mehr als 91\% Professoren bei insgesamt 34 Professuren.

Fachbereich VI: Informatik und Medien mit den Bachelor- und Master-Studiengängen Technische Informatik und Embedded Systems, Medieninformatik, Druck- und Medientechnik bei einem Anteil von $82 \%$ Professoren bei insgesamt 39 Professuren.

Fachbereich VII: Elektrotechnik - Mechatronik - Optometrie mit den Bachelor- und Master-Studiengängen Elektrotechnik, Mechatronik, Augenoptik/Optometrie, Energie- und Automatisierungssysteme bei einem Anteil von mehr als 95\% Professoren bei insgesamt 40 Professuren.

Sowie Fachbereich VIII: Maschinenbau, Veranstaltungstechnik, Verfahrenstechnik mit den Bachelor- und Master-Studiengängen Maschinenbau, Veranstaltungstechnik und -management, Verfahrens- und Umwelttechnik, Audiovisuelle Medien, Theatertechnik und Wirtschaftsingenieur (gemeinsame Studiengänge mit der HWR) bei einem Anteil von mehr als 82\% Professoren bei insgesamt 45 Professuren.

Die Tabelle 2 bietet einen Gesamtüberblick über die Fachbereiche und Berufungskorridore der Beuth Hochschule für Technik Berlin:

Bis 2022 werden an der Beuth Hochschule bei den Professuren 62 Stellen neu zu besetzen sein. Das entspricht rd. 20\% der Professuren und bietet erheblichen Spielraum für die Erreichung der Chancengleichheit bzw. der Gleichstellungsziele. Da die Zuweisung einer Professur zu einem Fachgebiet durch den Akademischen Senat nach kapazitiver Überprüfung erfolgt und Stelleninhaberinnen bzw. Stelleninhaber ihre Professuren zudem verlängern können, lässt sich das Freiwerden der Stellenpositionen nur ungefähr bestimmen. Dennoch ist es erforderlich, durch das frühzeitige in-den-Blick-Nehmen der potentiell freiwerdenden Positionen eine bessere Steuerung des Pools an potentiellen Bewerberinnen und Bewerbern anzustreben. Die Wiederbesetzung der Professuren eröffnet Chancen und Möglichkeiten, bestehende Geschlechterverhältnissen aufzubrechen ${ }^{15}$ und zu Gunsten der Frauen zu verändern. Gleichzeitig besteht die Gefahr, dass der Frauenanteil durch den Generationswechsel an der Beuth Hochschule weiter einbricht, da auch Professorinnen in den Ruhestand treten werden und diese neu entstehende Fehlstelle ebenfalls durch eine im Sinne der Gleichstellung der Geschlechter erfolgreiche Berufungspolitik geschlossen werden muss.

\begin{tabular}{|llr|}
\hline Fachbereiche der Beuth Hochschule für Technik Berlin & $\begin{array}{l}\text { Professorinnen } \\
\text { Anteil }\end{array}$ & $\begin{array}{l}\text { Freiwerdende } \\
\text { Professuren } \\
\text { bis 2022 }\end{array}$ \\
\hline $\begin{array}{l}\text { I: Wirtschafts- und Gesellschaftswissenschaften } \\
\text { Betriebswirtschaftslehre (Dual), Management und Beratung, } \\
\text { Wirtschaftsinformatik Online, Wirtschaftsingenieurwesen }\end{array}$ & $24 \%$ & 5 \\
\hline
\end{tabular}

\footnotetext{
${ }^{15}$ Verweis auf frühere Erhebungen z.B. zF-Berichte. Betrachten wir die Entwicklung der Professorinnen Anteile der letzten 10 Jahre, ist eine gewisse Stagnation der Geschlechterverteilung bei den Professuren erkennbar: Seit dem WS 2007/08 ist mit dem absoluten Anstieg der Professuren der Professorinnen Anteil auf 19\% gestiegen und nach einem „Ausreißer“-Anstieg im WS 2010/11 auf $20,6 \%$ nun 2016 wieder bei $19 \%$. Dies zeigt, wie fragil kurzzeitig erreichte Erfolge sein können, da nicht alle Neuberufenen dauerhaft an der Hochschule gehalten werden können.
} 


\begin{tabular}{|c|c|c|}
\hline $\begin{array}{l}\text { II: Mathematik - Physik - Chemie } \\
\text { Pharma- und Chemietechnik, Physikalische Technik - } \\
\text { Medizinphysik, Mathematik }\end{array}$ & $31 \%$ & 6 \\
\hline $\begin{array}{l}\text { III: Bauingenieur- und Geoinformationswesen } \\
\text { Bauingenieurwesen, Geoinformation, } \\
\text { Kartographie und Geomedien, Umweltingenieurwesen Bau, } \\
\text { Vermessungswesen und Geomatik }\end{array}$ & $9 \%$ & 7 \\
\hline $\begin{array}{l}\text { IV: Architektur und Gebäudetechnik } \\
\text { Architektur, Gebäude- und Energietechnik/ Energiemanagement, } \\
\text { Planung nachhaltiger Gebäude, Facility Management }\end{array}$ & $23 \%$ & 10 \\
\hline $\begin{array}{l}\text { V: Life Sciences and Technology } \\
\text { Biotechnologie, Gartenbauliche Phytotechnologie, } \\
\text { Landschaftsarchitektur, Lebensmitteltechnologie, } \\
\text { Urbanes Pflanzen- und Freiraum-Management, Verpackungstechnik }\end{array}$ & $30 \%$ & 10 \\
\hline $\begin{array}{l}\text { VI: Informatik und Medien } \\
\text { Technische Informatik und Embedded Systems, } \\
\text { Medieninformatik, Druck- und Medientechnik }\end{array}$ & $18 \%$ & 7 \\
\hline $\begin{array}{l}\text { VII: Elektrotechnik - Mechatronik - Optometrie } \\
\text { Elektrotechnik, Mechatronik, Augenoptik/Optometrie, } \\
\text { Energie- und Automatisierungssysteme }\end{array}$ & $5 \%$ & 8 \\
\hline $\begin{array}{l}\text { VIII: Maschinenbau, Veranstaltungstechnik, Verfahrenstechnik } \\
\text { Maschinenbau, Veranstaltungstechnik und -management, } \\
\text { Verfahrens- und Umwelttechnik, Audiovisuelle Medien, Theatertechnik }\end{array}$ & $18 \%$ & 9 \\
\hline
\end{tabular}

Tabelle 2: Fachbereichsspezifische Frauenanteile und Berufungskorridore

Ein Weg ist die Förderung und Akquirierung von Nachwuchswissenschaftlerinnen. Zwischen der Technischen Universität Berlin und der Beuth Hochschule besteht ein Kooperationsabkommen, das die Promotion für FH-Absolvierende erleichtern soll. In der Praxis unterliegen die Verfahren zur Aufnahme von FH-Absolvierenden als Promovierende der Technischen Universität Berlin der Einzelfallentscheidung der Fakultäten, die u.a. über mögliche Auflagen für die Aufnahme entscheiden. Wenn universitäre Studieninhalte innerhalb der Promotionsphase bzw. vor Promotionsannahme nachgeholt und nachgeholt werden müssen, ist dies weiterhin als Hürde zu bewerten, die den Promotionsverlauf zeitlich ungünstig beeinflussen kann.

Die Beuth Hochschule vergibt seit 2014 Promotionsstipendien an hochqualifizierte Absolventinnen und Absolventen staatlicher Berliner Fachhochschulen. Das Promotionsstipendium der Beuth Hochschule zielt darauf ab, FH-Absolvierende in der Abschlussphase ihre Promotion finanziell zu unterstützen. Die Ausgewählten erhalten für zwölf Monate ein Stipendium in Höhe von 1.250 Euro im Monat. Bewerben können sich Alumni aller Berliner Fachhochschulen mit einem sehr guten Abschluss und für deren Promotionsvorhaben bereits eine Zulassung an einer Berliner Universität vorliegt. Gewünscht - aber keine Bedingung - ist, dass die Bewerberinnen bzw. Bewerber in ihrem Promotionsvorhaben ein Forschungsthema bearbeiten, das im Zusammenhang mit der Hochschulstrategie „Stadt der Zukunft“ steht.

Ziel der Beuth Hochschule ist es, jedes Jahr zum Wintersemester vier Nachwuchswissenschaftlerinnen bzw. Nachwuchswissenschaftler neu in die Promotionsförderung aufzunehmen. Die Bewerbungsfrist endet jeweils am 30. Juni. Über die Vergabe der Stipendien entscheidet die Beuth-Forschungskommission. Gemeinsam betreut werden die Promovierenden durch je einen Professor oder eine Professorin der Beuth Hochschule und einer Berliner Universität. Die Forschungsarbeiten erfolgen an Standorten der Beuth Hochschule. In den drei Bewerbungsrunden von 2014 bis 2016 gingen sechs Bewerbungen von Nachwuchswissenschaftlerinnen und sechs von Nachwuchswissenschaftlern ein. Erfreulicherweise betrug der Frauenanteil an den 12 Bewerbungen damit $50 \%$. Von den eingegangenen Bewerbungen wurden insgesamt acht bewilligt. Bei der Vergabe dieser acht Stipendien hatten die Nachwuchswissenschaftlerinnen ebenfalls einen Anteil von 50\% (4 Stipendien). Unter den vier in die Beuth Promotionsförderung aufgenommenen FH-Absolventinnen waren zwei ehemals durch das Hypatia Programm ${ }^{16}$ geförderte.

\footnotetext{
${ }^{16}$ vgl. Teil B, S. 43
} 


\section{A2.2 Proaktive Rekrutierung}

Die fachliche Ausrichtung einer Professur ist maßgeblich dafür, ob überhaupt Bewerbungen von Wissenschaftlerinnen auf eine Ausschreibung eingehen. Die Analyse von 101 Berufungsverfahren in den Jahren 2010 bis 2015 an der Beuth Hochschule hat gezeigt, dass in $21 \%$ der Verfahren keine Bewerbung einer Frau vorlag ${ }^{17}$, in weiteren 40 Verfahren lagen max. drei Bewerbungen von Frauen vor. Dies macht deutlich, dass verstärkt das Augenmerk auf eine proaktive Rekrutierung von Interessentinnen gerichtet werden muss, wenn das Ziel erreicht werden soll, in jedem Berufungsverfahren auch Bewerbungen von Frauen zu erhalten.

\begin{tabular}{|crrr|}
\hline \multicolumn{4}{|c|}{ Berufungsverfahren ohne bzw. mit wenigen Bewerberinnen } \\
\hline Jahr & $\begin{array}{r}\text { Verfahren } \\
\text { insges. }\end{array}$ & $\begin{array}{c}\text { davon keine } \\
\text { Bewerberin }\end{array}$ & $\begin{array}{c}\text { davon max. drei } \\
\text { Bewerberinnen }\end{array}$ \\
\hline 2010 & 28 & 8 & 15 \\
2011 & 10 & 2 & 4 \\
2012 & 17 & 4 & 6 \\
2013 & 10 & 1 & 3 \\
2014 & 11 & 1 & 4 \\
\hline 2015 & 25 & 5 & 40 \\
\hline Summe & 101 & 21 & 8 \\
\hline
\end{tabular}

Tabelle 3: Frauenanteile nach Berufungsverfahren an der Beuth Hochschule

Die Berufungsverfahren dauern in der Regel deutlich länger als 1 Jahr, da nach Ruferteilung durch den Berliner Senat das Berufungsgespräch und Kündigungsfristen zu berücksichtigen sind. Mit der bisherigen Ausschreibungspraxis werden gerade in den technischen Fächern keine oder zu wenige Interessentinnen erreicht. Die Beuth Hochschule hat sich mit einem AS-Beschluss verpflichtet, regelhaft neu auszuschrieben, wenn keine einzige Bewerbung einer Frau eingegangen ist. Da dies den Kreis an Bewerberinnen nicht per se erweitert, hat die Hochschule versuchsweise in den letzten Jahren bei drei besonders schwierigen Verfahren gezielt externe Agenturen als sogenannte Headhunter eingeschaltet, die die aktive Suche nach geeigneten Bewerbenden unterstützt haben. Dabei erfolgt seitens der Agenturen nur die Identifikation und ggf. Erstansprache geeigneter Bewerberinnen. Dieser Zwischenschritt erhöht bestenfalls die Anzahl qualifizierter Bewerbungen, der Berufungsprozess wird unverändert durchgeführt.

Als besonders fragil hat sich bei diesem Vorgehen der Kommunikationsprozess zwischen Personalberatenden und Mitgliedern der Hochschule erwiesen: nur wenn dieser Verständigungsprozess eng und vertrauensvoll aufgesetzt werden kann, wird die Hochschule im Verfahren entlastet. Der zeitliche Aufwand für Berufungskommissionsmitglieder hingegen bleibt hoch und die Eigengesetzlichkeiten von Berufungsverfahren müssen den Personalberatenden zusätzlich detailliert erklärt werden. Unter Umständen gelingt allerdings im Idealfall eine Straffung des Gesamtverfahrens. Günstig ist es, bei Erfolg - z.B. der Platzierung einer Kandidatin auf der Berufungsliste - eine Kopfprämie für die Agentur auszuloben. Wie nachstehende Tabelle zeigt, liegt der Anteil von Frauen bei den Berufungen nah am Bewerberinnenanteil. Unter der Berücksichtigung der Prämisse „Frauen werden bei gleicher Qualifikation bevorzugt“ und vor dem Hintergrund der freiwerdenden derzeit noch mit Frauen besetzten Professuren muss der Frauenanteil bei den Berufungen allerdings deutlich steigen.

\footnotetext{
${ }^{17}$ Ausschreibungen ohne Bewerbung von Frauen werden standardmäßig erneut ausgeschrieben.
} 


\begin{tabular}{|lrrrr|}
\hline \multicolumn{5}{|c}{ Bewerbungen auf Professuren an der Beuth Hochschule für Technik Berlin } \\
\hline & gesamt & $\begin{array}{r}\text { Anteil- } \\
\text { W \% }\end{array}$ & Berufungen & $\begin{array}{r}\text { Anteil- } \\
\text { W \% }\end{array}$ \\
2010 & 691 & 14,5 & 18 & 22 \\
2011 & 366 & 20,8 & 22 & 18 \\
2012 & 317 & 14,8 & 14 & 14 \\
2013 & 284 & 23,6 & 17 & 24 \\
2014 & 346 & 24 & 10 & 20 \\
2015 & 741 & 24 & 16 & 25 \\
Gesamt & 2745 & 20 & 107 & 19 \\
\hline
\end{tabular}

Tabelle 4: Frauenanteile unter Bewerbungen auf Professuren der Beuth Hochschule

Eine weitere Möglichkeit, Bewerberinnen anzusprechen, stellt der Basisausschreibungstext der Beuth Hochschule dar. Auf Initiative der zentralen Frauenbeauftragten und in Zusammenarbeit mit dem GuTZ liegt der Hochschule ein neuer Entwurf vor, der eine zeitgemäße und attraktive Ansprache von Bewerberinnen gewährleisten könnte.

Aufgrund der Ergänzung „Interessierte Frauen können Kontakt zur zentralen Frauenbeauftragten der Hochschule aufnehmen“ haben potentielle Bewerberinnen in den vergangenen Jahren vermehrt die Frauenbeauftragte kontaktiert, vor allem bei Fragen, die sie nicht Fachvertreterinnen oder Fachvertretern stellen wollten. Deutlich wurde in diesen Kontakten, dass potentielle Bewerberinnen einen Perfektionismus entwickeln können, der eine hohe Hürde darstellt. Haben sie den Eindruck, dass sie sich nicht gut genug vorbereiten können, sehen sie von einer Bewerbung ab. Haben sie gerade eine große persönliche Umwälzung hinter sich, wie den Umzug mit Partnern und Kindern und den Neueinstieg in ein Unternehmen, sehen sie trotz Interesse von einer Bewerbung ab.

\section{A2.3 Hochschule im Wettbewerb}

Die Beuth Hochschule hat mit einer Repräsentanz von fast 81\% Männern auf Professuren einige Herausforderungen zu meistern, insbesondere, wenn sie ihre Frauenanteile an die positive Entwicklung der Hochschule für Wirtschaft und Recht HWR (2014 38\%) oder der Hochschule für Technik und Wirtschaft HTW (2014 29\%) angleichen möchte, die an Größe (Anzahl von Professuren und Studienplätzen) vergleichbaren Fachhochschulen in Berlin. Sind dort die Entwicklungen in den letzten Jahren als positiv zu bewerten, stagniert die Entwicklung an der Beuth Hochschule. Ähnlich verhält es sich bei den Frauenanteilen unter den Studierenden, die sich in den letzten zehn Jahren ebenfalls wenig weiterentwickelt haben. Vergleichbar zu unserer Hochschule verlief die Entwicklung an der Technischen Universität Berlin (2014, 20\% Anteil Professorinnen), wie in den Leistungsberichten der Hochschulen im Kapitel 6 „Gleichstellung“ sehr gut nachzuvollziehen ist (TUB 2015). Es liegt nahe, in diesen Stagnationen das Ergebnis eines klaren MINT-Profils zu vermuten.

Der praktischen Ausbildung kommt an der Beuth Hochschule enorme Bedeutung zu, Lehrbeauftragte aus der Industrie ergänzen daher das Lehrangebot entscheidend. Der Frauenanteil bei den Lehrbeauftragten an der Beuth Hochschule konnte von 24\% in 2013 auf $27 \%$ gesteigert werden (Wintersemester 2015: 591 Lehraufträge insgesamt, davon 160 Frauen).

Die Berliner Fachhochschulen müssen sich in der Berufungspraxis mit im Bundesdurchschnitt niedrigeren Leistungsbezügen in Konkurrenz zu Industrie, Universitäten und außeruniversitären Forschungseinrichtungen behaupten.

\section{A2.4 Stellenbesetzungen und Berufungen - bereits erprobte und erfolgreiche Maßnahmen}

Für die gezielte Suche nach geeigneten Bewerberinnen beteiligt sich die Beuth Hochschule an dem bereits oben genannten kooperativen Projekt PROfessur „Neue Chancen für Sie, für Frauen aus Wissenschaft und Praxis“, welches an der Hochschule in Hannover angesiedelt ist. ${ }^{18}$

\footnotetext{
${ }^{18}$ Gewinnung von Frauen für eine Fachhochschul PROfessur - Maßnahme der Landeskonferenz der niedersächsischen HochschulFrauenbeauftragten (LNHF), seit April 2002, http://professur.fh-hannover.de/index1.html
} 
Alle Ausschreibungen von Professuren laufen über die Datenbank bzw. den Verteiler dieses Projektes (es ist das Portal für den nord- und mitteldeutschen Raum). Die Webseite hält differenzierte Informationen zum Anforderungsprofil und Aufgabenspektrum einer Professur an einer Fachhochschule bereit. Darüber hinaus gibt es über diese Seiten u.a. das niedrigschwellige Angebot für Interessentinnen, Professorinnen bestimmter Fachrichtungen als Expertinnen vor Ort per Email zu kontaktieren.

Mit einer kooperativen Veranstaltung im Deutschen Technikmuseum, ${ }^{19}$ gemeinsam mit dem Verein Deutscher Ingenieure VDI Berlin-Brandenburg und der HTW Berlin, ist die Beuth Hochschule 2014 verstärkt im Berliner Raum in die Offensive gegangen, um für die Karriere „Professorin an einer Hochschule im MINT-Bereich werden“ z.B. mit Rollenvorbildern zu werben. Die Veranstaltung war mit 80 Teilnehmerinnen sehr gut nachgefragt. Im Juli 2016 wurde ein ähnliches Format in Kooperation ${ }^{20}$ mit HTW Berlin und HWR Berlin fortgeführt, mit dem erneut 80 Teilnehmerinnen erreicht werden konnten. Inhaltlicher Schwerpunkt waren 2016 die formalen Voraussetzungen im Berufungsverfahren. Zielgruppe dieser Veranstaltungsreihe sind bereits qualifizierte Wissenschaftlerinnen, die sich auf in Kürze ausgeschriebene Stellen bewerben könnten. Aus den Diskussionen und Gesprächen der Veranstaltung lässt sich schließen, dass es vor allem bei den Wissenschaftlerinnen, denen noch ein Karrierebaustein fehlt - entweder die Praxisphase oder die Promotion - ein reges Interesse an der FH-Professur besteht.

Gemeinsam haben die drei beteiligten Hochschulen in den nächsten 5 Jahren ca. 160 Stellen zu besetzen, davon allein ca. 60 Stellen an der Beuth Hochschule. Aus diesem gemeinsamen Interesse heraus wird auch 2017 an diesem Veranstaltungsformat festgehalten.

\section{A2.5 Gleichstellungskonzept der Beuth Hochschule}

Die Beuth Hochschule fördert die Verwirklichung der Gleichstellung von Frauen und Männern und wirkt auf die Beseitigung bestehender Nachteile hin. Dazu gehören der Abbau struktureller Hemmnisse zur Erreichung der Chancengleichheit, die Erhöhung der Anteile des jeweils unterrepräsentierten Geschlechts sowie die Integration von Genderaspekten in Lehre und Forschung.

Die Familienfreundlichkeit ist ein integraler Bestandteil. Die Beuth Hochschule sieht sich in der Verantwortung, eine tatsächliche Vereinbarkeit von Studium, Beruf und Familie zu gewährleisten. Sie fördert die Diversität ihrer Studierenden (38,8\% haben eine weitere nichtdeutsche Muttersprache, Erstsemesterbefragung Sommersemester 2015) und ermöglicht ein Studium mit Behinderung.

Erstmalig hat die Beuth Hochschule seit 2013 ein Gleichstellungskonzept, welches gemeinsam mit den Frauenförderrichtlinien und der Satzung „Chancengleichheit der Geschlechter“ (2014) neue Durchsetzungsmöglichkeiten für die Gleichstellung bietet. 2015 hat das Gleichstellungskonzept eine Aktualisierung im Hinblick auf die Schärfung der Gleichstellungsstrategie der Hochschule erfahren. ${ }^{21}$

Die geplanten Maßnahmen orientieren sich an vier strategischen Zielen und werden als Querschnitt in vier Handlungsfelder aufgeteilt: Nachwuchsförderung, Personalmanagement, Vereinbarkeit und Lehre und Forschung, vgl. Tabelle 5.

Inhouse-Schulungen zum Thema Berufungsverfahren sind an der Beuth Hochschule seit Jahren etabliert. Im Zuge der Ergebnisse der Berufungsverfahren fand eine inhaltliche Anpassung statt.

\footnotetext{
${ }^{19}$ Wie werde ich Professorin einer technischen Fachhochschule? - Fachtagung der Beuth Hochschule am 5. 11.2014. Link: http://m.komm-mach-mint.de/MINT-News/Partner-News/Beuth-Hochschule-Fachtagung-Professorin

${ }^{20}$ Berufsperspektive: Professorin an einer Hochschule - Qualifikationsanforderungen und Berufungsverfahren. Vortrag und Workshops, 14.07.2016

${ }^{21}$ Gleichstellungskonzept der Beuth Hochschule für Technik Berlin, http://www.beuth-hochschule.de/de/3710/
} 


\begin{tabular}{|cc|}
\hline \multicolumn{1}{|c|}{ Strategisches Ziel } & Handlungsfeld \\
\hline $\begin{array}{c}\text { Erweiterung des Pools von Bewerberinnen } \\
\text { und höhere Berufungsquote von Profes- } \\
\text { sorinnen in MINT-Fächern }\end{array}$ & $\begin{array}{c}\text { Nachwuchsförderung und } \\
\text { Personalmanagement }\end{array}$ \\
$\begin{array}{c}\text { Erhöhung des Anteils von Studentinnen für } \\
\text { MINT-Fächer }\end{array}$ & $\begin{array}{c}\text { Nachwuchsförderung und } \\
\text { Vereinbarkeit }\end{array}$ \\
$\begin{array}{c}\text { Chancengerechte strukturbildende Maß- } \\
\text { nahmen auf allen Ebenen und für alle } \\
\text { Statusgruppen der Hochschule }\end{array}$ & $\begin{array}{c}\text { Vereinbarkeit und } \\
\text { Kulturwandel in Forschung und Lehre in }\end{array}$ \\
MINT-Fächern durch Integration von Gen- \\
der und Diversity
\end{tabular}

Tabelle 5: Strategische Ziel und Handlungsfelder der Gleichstellung

2014 fand als eine Maßnahme des Gleichstellungskonzeptes unter der Leitung des Gender- und Technik-Zentrums (GuTZ) der Beuth Hochschule in Person von Frau Prof. Dr. Antje Ducki sowie unter Mitwirkung der Frauenbeauftragten erstmals eine Schulung von Berufungskommissionsmitgliedern zum Thema gender- und diversitygerechte Personalauswahl mit hochschuleigenem Konzept statt. Neben verwaltungsrechtlichen Aspekten des Berufungsvorgangs stand eine diskriminierungsfreie Standardisierung des Verfahrens im Mittelpunkt der Schulung. Ab 2016 werden pro Jahr mindestens zwei Durchgänge angeboten. Diese Veranstaltung ist eingebettet in ein umfassendes Personalmarketingkonzept, das verstärkt Frauen adressieren soll.

\section{A2.6 Durchlåssigkeit auch in Karrierephasen fördern}

Wie das Leitbild der Beuth Hochschule verdeutlicht, sieht sich die Hochschule in der IngenieurTradition ihrer Gründungsväter verankert, legt dabei aber ein großes Gewicht auf die Weiterentwicklung und das Erreichen der Chancengleichheit von Frauen und Männern.

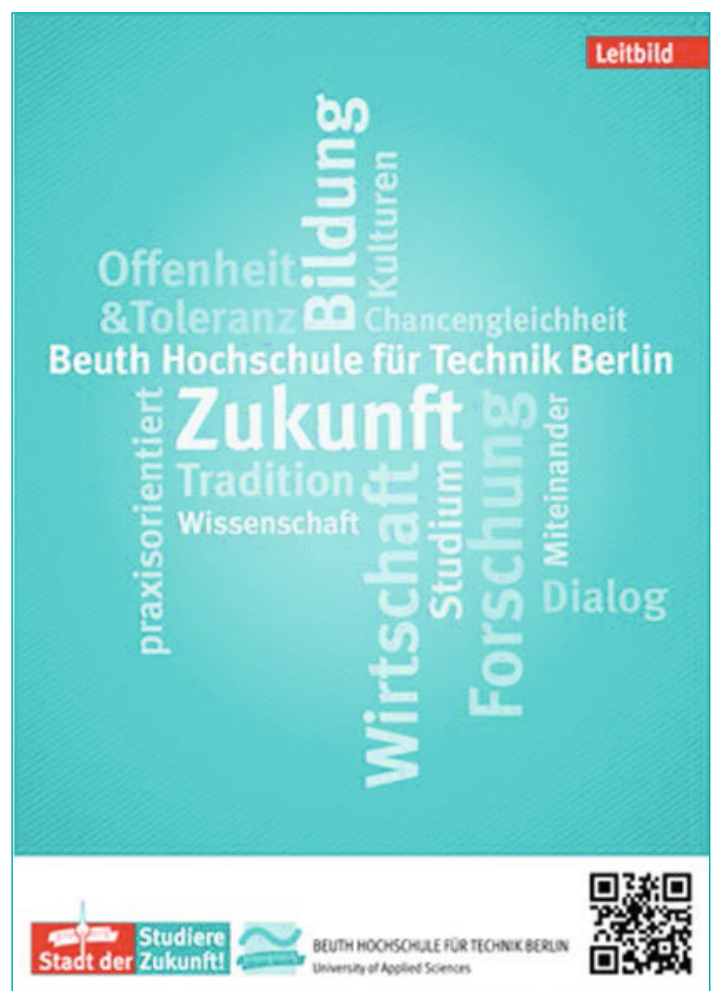

Abbildung 2: Leitbild der Beuth Hochschule für Technik Berlin 
Da das Fächerprofil deutlich MINT-geprägt ist, mit höchster Absolvierendenzahl im Ingenieurbereich für Berlin, stellt es damit weiterhin eine große Herausforderung für die Hochschule dar, auch für ein Studium, Studieninteressentinnen zu gewinnen. „Die geringen und längerfristig betrachtet stagnierenden Quoten weiblicher Studienanfänger in der Gruppe der technischen Studienfächer können dabei als zentraler Indikator für eine nach wie vor geschlechtsspezifische Segregation in den fachkulturellen Orientierungen gelten." (Wensierski et.a. 2015, S. 17)

Nachwuchsförderung beginnt mit dem Studium. Nach wie vor gilt auch für Fachhochschulen eine ausgeprägte geschlechtsspezifische Studienfachwahl. Das durch die Gleichstellungsarbeit über Jahrzehnte kontinuierlich verfolgte Ziel der Erhöhung des Studentinnen Anteils in MINT Studienfächern muss weiterhin verfolgt und gefördert werden.

Die Kooperationen zwischen Universitäten und Fachhochschulen im Bereich von Promotionen müssen weiterhin gestärkt und verbessert werden. Dies dürfte unter Gleichstellungsgesichtspunkten im Interesse der Universitäten sein, da die Fachhochschulen talentierte Nachwuchswissenschaftlerinnen hervorbringen und damit den kleinen Pool im Ingenieurbereich anreichern. Hieran knüpft sich aber auch die Diskussion über ein eigenständiges Promotionsrecht von Fachhochschulen für einschlägige FH-Studiengänge an sowie die Ermöglichung eines wissenschaftlichen Mittelbaus an Fachhochschulen. Für Anstrengungen in diesem hochkomplexen Feld gilt grundsätzlich, dass sie mit geschlechtergerechten Qualitätsstandards und personellen Zielgrößen insbesondere zur Erhöhung der Frauenanteile auf Führungspositionen in beiden Hochschultypen untrennbar verknüpft sein müssen.

Grundsätzlich sind planbare Karrierewege vorteilhaft für die Gewinnung und den Verbleib von Frauen im Wissenschaftssystem. In der globalen Debatte über „verstopfte Karrierewege für den wissenschaftlichen Nachwuchs“ hat Prof. Dr. Bernd Reissert im Rahmen der Hochschulallianz $\mathrm{UAS}^{22}$ zu Recht darauf hingewiesen:

„In der Debatte wird meist übersehen, dass die Professuren an den Universitäten nur $53 \%$ aller Professuren in Deutschland ausmachen. 41\% der Professuren sind Professuren an Fachhochschulen." ${ }^{23}$

${ }^{22}$ Der Zusammenschluss von sieben forschungsorientierten deutschen Fachhochschulen mit starker internationaler Ausrichtung stellt sich vor, http://www.uas7.de/

${ }^{23}$ Prof. Dr. Bernd Reissert im Rahmen der Hochschulallianz UAS7,

http://www.uas7.de/fileadmin/Dateien/UAS7_wissenschaftlicher_Nachwuchs.pdf 


\section{A3 Gewinnung von FH-Professorinnen im MINT-Bereich}

\section{A3.1 Projekte im Rahmen des BCP}

Im Rahmen des Berliner Chancengleichheitsprogramm BCP wurden mit dem in einem Zusammenschluss von 13 der 14 Hochschulen in Berlin beantragten und an der Technischen Universität Berlin angesiedelten Graduiertenprogramm mit dem Themenschwerpunkt „Digitalisierung“ neue Formen der Kooperation und Frauenförderung etabliert: geplant sind Ausschreibungen von Promotionsstellen für Fachhochschulabsolventinnen und Postdoc-Stellen an Universitäten noch in $2017 . .^{24}$

Die Beuth Hochschule hat 2015 die Ausschreibung des Berliner Programms für Chancengleichheit von Frauen genutzt und neben der Einwerbung von Gastdozenturen bzw. Gastprofessuren und vorgezogenen Nachfolgeberufungen ab 2016 drei neue Projektideen gestartet, die genau da ansetzen, wo bisherige Förderungen u.a. im Rahmen des Hypatia Programms aufhörten:

\section{Entwicklung einer „Place to be“ Strategie zur Gewinnung von FH-Professorinnen im MINT- Bereich}

Ziel ist es, Fachkulturen für Frauen weiter zu öffnen sowie langfristige fachspezifische Berufungsstrategien zur Gewinnung von MINT Professorinnen zu entwickeln, um die Arbeitgeberinnenattraktivität der Hochschule zu verbessern (place to be - Strategie). Eine gender- und diversitygerechte Berufungspraxis wird in die langfristige Entwicklung der Fächergruppen integriert. Mittelfristig sollen in den einzelnen Fachgebieten die Voraussetzungen für vorgezogene Nachfolgeberufungen verbessert werden. Die Unterstützung der Fachbereiche mit geringem Frauenanteil bei der Entwicklung langfristiger Berufungsstrategien erfolgt durch Abstimmung der freiwerdenden Stellen (Berufungskorridore) mit zukünftigen fachlichen Innovationspotentialen, Ableitung von Anforderungsprofilen und fachspezifischen Akquise Strategien vorrangig im IT- und Ingenieurbereich.

Zur Umsetzung dient die Entwicklung und Erprobung eines gendersensiblen E-Learning-Formats „Berufungsschulung“ für die Zielgruppe der Berufungskommissionsmitglieder. Dies könnte gerade unter dem Aspekt der hohen Lehrbelastung nachhaltig sicherstellen, dass alle Mitglieder einer Berufungskommission zum Zeitpunkt der aktuellen Personalauswahl für eine gender- und diversitygerechte Personalauswahl sensibilisiert und qualifiziert sind. Das E-Learning-Angebot kann darüber hinaus in Teilen anderen Hochschulen zugänglich gemacht werden und dient damit auch einer Arbeitgeberinnenpositionierung als Hochschule, die aktiv bestrebt ist, Professorinnen zu rekrutieren. Mit der Entwicklung und Erprobung eines ergänzenden blended E-Learning-Angebots zum Thema „Professioneller Auftritt in Berufungsgesprächen“ für potentielle Bewerberinnen bietet sich neben der Einzelförderung auch die Möglichkeit, Interessentinnen stärker an sich zu binden. Damit sollen die Wissenschaftlerinnen gerade in Minderheitenpositionen gestärkt werden, um einem möglichen Genderbias in der Personalauswahl auf Seiten der Bewerberinnen wirksam begegnen zu können.

\section{Erschließung neuer Zielgruppen für Professuren im MINT-Bereich}

Ziel ist die Erhöhung der Anzahl der Professorinnen im MINT-Bereich durch Erschließung internationaler Zielgruppen und Frauen mit fehlenden formalen Berufungsvoraussetzungen.

Notwendig hierzu ist der Aufbau internationaler Kontakte und Netzwerke zur Bewerberinnenrekrutierung außerhalb Deutschlands. Kontakte zu internationalen Netzwerken und Jobbörsen bestehen an der Beuth Hochschule bislang nicht. Besonderheiten des deutschen Bildungssystems hinsichtlich der Anforderungen und Stellung der Fachhochschulen müssen zielgruppengerecht aufbereitet werden. Dies soll auch anhand eines zweisprachigen Webauftritts geschehen. Anforderungen und Aufgabenstellung, aber auch Entwicklungspotentiale für potentielle Bewerberinnen müssen deutlich gemacht werden. Ggf. können geflüchtete Akademikerinnen als weitere Zielgruppe rekrutiert werden.

${ }^{24}$ Die Ausschreibungen werden über die Seiten der Technischen Universität Berlin veröffentlicht. 
Die Entwicklung und Erprobung von Veranstaltungsformaten für potentielle Bewerberinnen, denen formale Voraussetzungen zur FH-Professur fehlen (Anpassungs- und Weiterqualifikation, berufsbegleitende Promotionen in Kooperation von Wirtschaft und Hochschule, Fernstudien-Angebote zur Aktualisierung des im Studium erworbenen Fachwissens, berufsbegleitende Weiterbildungsformate sowie Seminare für Promovierende). Für Bewerberinnen aus dem Inland, Geflüchtete, Bewerberinnen aus dem Ausland müssen entsprechende Veranstaltungen angeboten werden, die jeweils auf die Zielgruppen angepasst werden müssen.

Die Entwicklung, Erprobung sowie Durchführung eines Motivationstrainings soll - in Anlehnung an das Führungsmotivations-Coaching (FÜMO) der Helmut-Schmidt-Universität Hamburg ${ }^{25}$ - für formal geeignete Wissenschaftlerinnen mit Praxiserfahrung und promovierte Ingenieurinnen aus Industrie und Wirtschaft erfolgen. Erstmalig soll dieses Training auf die Hochschulkarriereplanung angepasst und mit geeigneten Kandidatinnen durchgeführt werden.

\section{Hochschulkarriere für FH-Absolventinnen aus bildungsfernen Schichten, speziell mit Migrationshintergrund}

Die Erweiterung des Bewerberinnenpools für Professuren im MINT-Bereich auf Alumni und Masterabsolventinnen mit FH-Abschluss muss bereits frühzeitig ansetzen. Gelingen soll dies durch die Ausarbeitung eines Förderprogramms zum frühzeitigen Motivationsaufbau für Masterabsolventinnen und zur Weiterqualifizierung von Alumni der Beuth Hochschule zur FH-Professur. Da ca. 39 \% der Studierenden der Beuth Hochschule angeben, eine zweite Muttersprache neben Deutsch zu sprechen, soll ein Förder- und Entwicklungskonzept ausgearbeitet werden, das Absolventinnen und Alumni mit Migrationshintergrund aus bildungsfernen Schichten adressiert. Dazu sind im Einzelnen folgende Maßnahmen geplant:

Identifikation geeigneter Masterstudentinnen und Alumni der Beuth Hochschule insbesondere mit Migrationshintergrund, z.B. durch Empfehlungen der Studiengangsleitungen, oder aus dem Pool ehemaliger durch das Hypatia Programm geförderter Frauen sowie aus dem neuen Beuth Stipendienprogramm.

Daran anschließend soll die Analyse der Karrierevorstellungen und Rahmenbedingungen der Zielgruppe und damit Ableitung förderlicher Unterstützungsmaßnahmen eines Förderprogramms erfolgen.

Differenzierte Ausarbeitung des Gesamtprogramms auf Basis der Analyseergebnisse mit den (Etappen)-Zielen Vervollständigung insbesondere industrieller bzw. wirtschaftlicher Berufserfahrung, Promotion und Lehrerfahrung entspr. $\$ 100$ BerlHG bis zur Professur. Durchführung von Karriere-Beratungen und Karriereschulungen der identifizierten qualifizierten Studentinnen und Alumni und Unterstützung bei der Suche nach geeigneten Förderfonds und Finanzierungsmöglichkeiten einer Promotion.

\section{A3.2 Personalentwicklung als Zukunftsfeld}

All diese Aktivitäten und Maßnahmen an der Beuth Hochschule und in den eingegangenen Kooperationen sind Bausteine einer Personalentwicklung zur Erhöhung des Anteils des weiblichen wissenschaftlichen Nachwuchses.

Die Beuth Hochschule wird sich mittelfristig der Herausforderung stellen müssen, ihre Einzelaktivitäten in ein Gesamtkonzept zu überführen, wenn sie von weiteren Förderprogrammen für HAW profitieren möchte. Die Empfehlungen der HRK vom Oktober 2016 legen dar, dass zentrales Element ein konzeptbasiertes Antragsverfahren sein wird, in dem die einzelnen Hochschulen ihre Strategie und die daraus abgeleiteten Maßnahmen zur Gewinnung von Professorinnen und Professoren konkretisieren: „Wesentlicher Bestandteil ist ein Professorinnen- und Professoren-Gewinnungskonzept, welches an den spezifischen Bedürfnissen und Problemlagen der Hochschule ausgerichtet ist. Vorausgesetzt wird, dass Personalentwicklung für das gesamte wissenschaftliche Personal ein strategisches Handlungsfeld der Hochschulleitung ist und sie über ein Personalentwicklungskonzept verfügt, das Aussagen zu Standards, zum Grad der ins-

${ }^{25}$ https://web.hsu-hh.de/fak/geiso/fach/psy-aow 
titutionellen Verankerung und Stand der Umsetzung enthält.“ (HRK 2016)

Im Rahmen der Einstein Stiftung Digital Future ${ }^{26}$ hat die Beuth Hochschule zwei befristete Professuren eingeworben, die als „Profilprofessuren“ eingerichtet werden könnten. Zeitgleich läuft ein Auswahlverfahren zu einer vorgezogenen Nachfolgeberufung ebenfalls in der Informatik.

Innerhalb der Berliner Qualitäts- und Innovationsoffensive 2016-2020 setzt die Hochschule ein Programm für Teilzeit-Gastdozenturen mit einer Lehrbelastung von durchschnittlich 9 SWS auf, welches Wissenschaftlerinnen und Wissenschaftler aus der Praxis den berufsbegleitenden Erwerb von hochschuldidaktischen Kompetenzen ermöglichen wird, um deren Chancen und deren Interesse an einer Professur an der Beuth Hochschule zu erhöhen.

Mit diesem Maßnahmenbündel befindet sich die Hochschule auf dem richtigen Weg.

${ }^{26}$ Schaffung von 50 neuen Professuren im IT-Bereich, http://be-digital.berlin/ 
Auswertung und Fazitzum Hypatia Programm derBeuth Hochschule-22 JahreFörderung von Wissenschaftskarrieren herausragender MINT-Absolventinnen der Beuth Hochschule und ingenieurwissenschaftlicher Nachwuchswissenschaftlerinnen Ursula Diallo-Ruschhaupt

\section{B1 Programmstruktur zur Nachwuchsförderung bis 2015}

\section{B1.1 Einleitung}

Zur Erhöhung des Anteils des weiblichen wissenschaftlichen Nachwuchses in Lehre und Forschung sowie zur Verbesserung der Berufseinstiegschancen und -möglichkeiten von Absolventinnen hat die Beuth Hochschule für Technik Berlin (Beuth Hochschule) ehemals Technische Fachhochschule Berlin (TFH) - wie vorstehend bereits ausgeführt - vielfältige Einzelaktivitäten konzipiert und umgesetzt.

In den Archivalien der TFH aus dem Jahre 1992 finden sich zum Beispiel Dokumente zu einer erfolgreichen Umsetzung von allgemeinen Frauenfördermaßnahmen und personengebundenen Fördermaßnahmen, die maßgeblich von engagierten Frauen der Hochschule auf den Weg gebracht und begleitet wurden. Die Historie des Hypatia Programms ist ausführlich dokumentiert (FFK 2004). Für eine Professionalisierung und Etablierung der zu diesem Zeitpunkt an der TFH bestehenden personengebundenen Förderung von Frauen setzten sich zwei Jahre später, 1994, die Frauenkommission als Forschungskommission des Akademischen Senats und das Plenum der Frauenbeauftragten ein. Mit Erfolg: Das Hypatia Programm zur Förderung des weiblichen wissenschaftlichen Nachwuchses wurde gegründet und die bestehende personengebundene Förderung auf die „Förderung von Promotionsvorhaben von Absolventinnen der Hochschule“ ausgeweitet. Die rechtlichen Rahmenbedingungen, die FH-Absolvierenden den Weg zur Promotion eröffneten, hatte die Kultusministerkonferenz (KMK) mit dem Beschluss zur Öffnung der Universitäten für Promotionen von besonders qualifizierten FachhochschulAbsolvierenden ${ }^{27}$ im Dezember 1992 geschaffen (HRK 2007, S. 22).

Im Rahmen des Hypatia Programms hat die Beuth Hochschule von 1994 bis 2015 aktiv Wissenschaftskarrieren herausragender MINT-Absolventinnen der Hochschule und MINT-Nachwuchswissenschaftlerinnen gefördert.

Übergeordnetes Ziel der vielfältigen Fördermaßnahmen des Hypatia Programms war, Absolventinnen der Hochschule und Frauen mit natur- und ingenieurwissenschaftlichen Abschlüssen für Berufswege in Lehre und Forschung zu motivieren und weiter zu qualifizieren. Zur Zielerreichung wurden an Absolventinnen der Hochschule Stipendien zur Vorbereitung (bis 2009) und Durchführung von Promotionsvorhaben sowie an Nachwuchswissenschaftlerinnen der MINT-Fächer Gastdozenturen und Gastprofessuren vergeben.

Ein weiterer Förderschwerpunkt war bis 1997 die Einbindung von Absolventinnen in Forschungsprojekte der Hochschule, um ihre Berufseinstiegschancen zu verbessern.

Mit Blick auf die neue Studien- und Abschlussstruktur der Hochschulen mit Bachelor und Master unterstreicht die Hochschulrektorenkonferenz (HRK) in ihrer Empfehlung vom 10.02.2004, also zwölf Jahre nach dem Beschluss der KMK zur Öffnung der Universitäten für Promotionen von besonders qualifizierten Fachhochschul-Absolvierenden, die Durchlässigkeit zwischen den Hochschultypen für Promotionen und betont:

„Die Zulassungsentscheidung [zur Promotion, Anmerkung der Autorin] muss auf der Grundlage der fachlichen Qualifikationen des Bewerbers getroffen werden. Eine rein formale Auswahl, die auf einer Differenzierung nach Hochschultypen basiert, ist mit der Zielsetzung der gestuften Studienstruktur nicht vereinbar und im Interesse der gewünschten Durchlässigkeit nicht ak-

\footnotetext{
27 Promotionszugang für besonders qualifizierte Fachhochschulabsolventen, Beschluss der Kultusministerkonferenz vom 3./4.12.1992 i. d. F. vom 16.12.1994

bzw. Gastprofessorinnen des Hypatia Programms sowie Gender Gastprofessorinnen aus.
} 
zeptabel.“ (HRK 2007, S. 25)

Die formale Gleichbewertung von Master-Abschlüssen der Universitäten und Fachhochschulen als Zugangsberechtigung zur Promotion wurde durch diese Empfehlung der HRK deutlich unterstrichen.

In der Praxis mussten besonders qualifizierte Fachhochschul-Absolvierende mit einem Diplomabschluss an Universitäten durchweg zusätzliche Leistungsnachweise erbringen, um als Promovierende aufgenommen zu werden. Dies zeigen die Förderverläufe der Hypatia Promovendinnen. Und auch nach 2004 ist die formale Gleichstellung von Universitäts- und Fachhochschul-Masterabschlüssen in der Praxis nicht voll umgesetzt.

Zusammenfassend ist festzuhalten, dass Fachhochschul-Absolvierende gerade erst einmal seit rund 20 Jahren die Möglichkeit haben, sich mit ihrem erworbenen Studienabschluss durch eine Promotion wissenschaftlich weiter zu qualifizieren und vor gut 10 Jahren von der HRK deutlich betont wurde, dass im formalen Zulassungsverfahren zur Promotion Masterabschlüsse von FH-Absolvierenden wie die der Universitätsabsolvierenden zu bewerten sind.

Deshalb ist es bedauerlich, dass das BCP in den Richtlinien des Förderzeitraumes 2016 bis 2020 die besondere finanzielle Förderung von Promotions-Programmen für FH-Absolventinnen, die an Berliner Hochschulen bestanden, beendet hat und stattdessen den Aufbau eines Graduiertenkolleg mit Schwerpunkt Digitalisierung verfolgt. Dies zu einem Zeitpunkt, zu dem eine strukturelle Verankerung und Ausgestaltung einer Promotionskultur im Sinne einer Fachkultur an der Beuth Hochschule gerade erst auf den Weg gebracht worden ist. Zum Beispiel werden an der Beuth Hochschule erste Mittel zur Promotionsförderung von Absolvierenden der Hochschule bereitgestellt ${ }^{28}$ und wurden erste Diskussionen über eine Institutionalisierung der Promotionsförderung geführt.

\section{B1.2 Hypatia Programm}

Die Maßnahmen des Hypatia Programms wurden seit der Gründung durchweg aus Drittmitteln finanziert. Von 1994 bis 2000 standen für das Hypatia Programm Mittel aus dem Hochschulsonderprogramm II (HSP II) bzw. dem Hochschulsonderprogramm III (HSP III) zur Verfügung. Das HSP lief Ende 2000 aus. Ab 2001 bis 2015 beantragte die Beuth Hochschule für das Hypatia Programm dann Mittel aus dem quotierten Bereich des Berliner Programms zur Förderung von Frauen in Forschung und Lehre (BCP). Aufgrund der durchgängigen Mittelbewilligung konnte das Hypatia Programm von 1994 bis Ende 2015 inhaltlich erfolgreich weiterentwickelt werden.

Alle im Rahmen des Hypatia Programms geförderten Maßnahmen wurden von 2001 bis 2009 durch fachübergreifende Seminar- und Workshop-Angebote begleitet und unterstützt, die ebenfalls aus Mitteln des BCP finanziert wurden. Für dieses Rahmenprogramm wurden ab 2010 aus dem BCP keine Mittel mehr bewilligt. Weitergeführt wurde bis Ende 2015 ein Mentoring- und Individualcoaching-Angebot.

Die Auswahl der Geförderten lag während der gesamten Programmlaufzeit verantwortlich bei der FrauenFörderKommission ${ }^{29}$ der Beuth Hochschule.

Die nachstehenden Ausführungen konzentrieren sich auf eine qualitative und quantitative Dokumentation und Bewertung der Fördermaßnahmen und Rahmenbedingungen des Hypatia Programms von 2001 bis 2015, also auf den Zeitraum, in dem Maßnahmen des Hypatia Programms voll aus BCP-Mitteln finanziert wurden.

\footnotetext{
${ }^{28}$ vergl. Abschnitt A, II. Zur aktuellen Situation an der Beuth Hochschule. Berufungskorridore, S. $11 \mathrm{f}$

${ }^{29}$ Die FrauenFörderKommission (FFK) ist eine Kommission des Präsidiums. Sie ist das Beschluss fassende Gremium für die Vergabe von personengebundenen Mitteln aus dem Berliner Programm zur Förderung der Chancengleichheit für Frauen in Forschung und Lehre. In dieser Funktion begutachtet die FFK eingegangene Bewerbungen und wählt die Stipendiatinnen und Gastdozentinnen
} 


\section{B1.2.1 Veränderungen des Förderkonzepts in Anpassung an Veränderungen der Finanzierungs-}

mo̊glichkeiten bzw. des Finanzierungsrahmens

Die Finanzierung von Maßnahmen des Hypatia Programms erfolgte von 2001 bis 2015 wie eingangs bereits erwähnt - insgesamt aus quotierten Mitteln des BCP. Andere Drittmittel wurden nicht akquiriert und Haushaltsmittel der Beuth Hochschule standen nur in Ausnahmesituationen ergänzend zur Verfügung. Diese enge Bindung an die Mittelzuweisung durch das BCP hatte zur Folge, dass die Vergaberichtlinien des BCP maßgeblich die Finanzierungsmöglichkeiten bzw. den Finanzierungsrahmen für das Hypatia Programm vorgaben und das Förderkonzept des Hypatia Programms an die BCP-Richtlinien angepasst werden musste.

Diese Anpassungsleistungen lassen sich in drei Phasen einteilen.

Phase 1 (2001 bis 2009): Der Maßnahmenkatalog, der gemäß den BCP Richtlinien förderungswürdig war, wurde in dieser ersten zeitlichen Phase vielfältig erweitert. Neben Mitteln für Promotionsstipendien und Gastdozenturen wurden Mittel für den Bereich Qualifizierung und Coaching, für Lehraufträge und Forschungsfreistellungen zur Verankerung von Genderaspekten in der Lehre und Forschung sowie als Pilotprojekt Mittel für ein Mentoring-Programm bewilligt.

Phase 2 (Mittelaufstockung in den Jahren 2008/2009 und Antragsrunde 2010/2011): 2008 und 2009 leitete das BCP mit einer Mittelaufstockung zur Finanzierung von Gastdozenturen und Gastprofessuren als Post Doc Förderung eine Neuorientierung der Mittelbewilligungspolitik ein. Von der zusätzlichen Bewilligungsrunde blieben die bereits seit 2007 laufenden und bis Ende 2009 bewilligten Maßnahmen unberührt.

In der Antragsrunde für die Förderjahre 2010 und 2011 griff dann die veränderte BCPMittelbewilligung. Anträge auf Mittelzuweisung für Sach- und Personalmittel zur Durchführung von begleitenden Maßnahmen zum Hypatia Programm wurden vom BCP nicht mehr bewilligt. Für die Förderung von Beuth Absolventinnen zur Promotion standen somit ab 2010 ausschließlich BCP-Mittel für die Stipendienvergabe zur Verfügung. Das Konzept der personengebundenen Förderung des Hypatia Programm musste entsprechend novelliert werden.

Dem GuTZ gelang es, aus dem Beuth Haushalt Mittel zu akquirieren und ein Teilangebot des ehemaligen fachübergreifenden Seminar- und Workshops-Angebots über das Fernstudieninstitut zu sichern.

Das Hypatia Programm nahm die veränderte Mittelbewilligungspolitik des BCP zum Anlass die Vergabe von Hypatia Gastdozenturen und Hypatia Gastprofessuren neu zu strukturieren. Ziel wurde es, über die Vergabe insbesondere von Gastprofessuren Gender- und Diversitythemen in der Lehre noch deutlicher zu stärken und neue innovative Lehrgebiete an der Beuth Hochschule zu verankern.

Phase 3 (2012 bis 2015): Bereits im Vorfeld der Mittelplanungen und -beantragung für den Förderzeitraum 2012 bis 2015 signalisierte das BCP, das Mittel für Promotionsstipendien absehbar nicht mehr bereit gestellt würden. Die Entscheidung, die Finanzierung von Promotionsstipendien aus BCP Mitteln mittelfristig einzustellen, wurde zu Beginn der Förderrunde $2012 / 2015$ bereits von wissenschafts- und hochschulpolitischen Gremien vorbereitet und erste Veränderungen umgesetzt.

Für den Förderzeitraum 2012 bis 2015 sahen die BCP Richtlinien nur noch die Bewilligung von Stipendien zur Förderung von Promotionen an Fachhochschulen vor, gleichzeitig wurde eine Verringerung des Mittelansatzes für Hypatia Promotionsstipendien eingeläutet.

Mit Beginn der Förderrunde 2016/2020 werden hochschulspezifische Promotionsprogramme an Fachhochschulen aus BCP-Mitteln nicht mehr gefördert. 


\section{B1.2.2 Förderzeitraum 2001 bis 2015}

Von 2001 bis 2015 wurden aus Mitteln des Hypatia Programms insgesamt 77 Fördermaßnahmen für 75 Frauen $^{30}$ bewilligt.

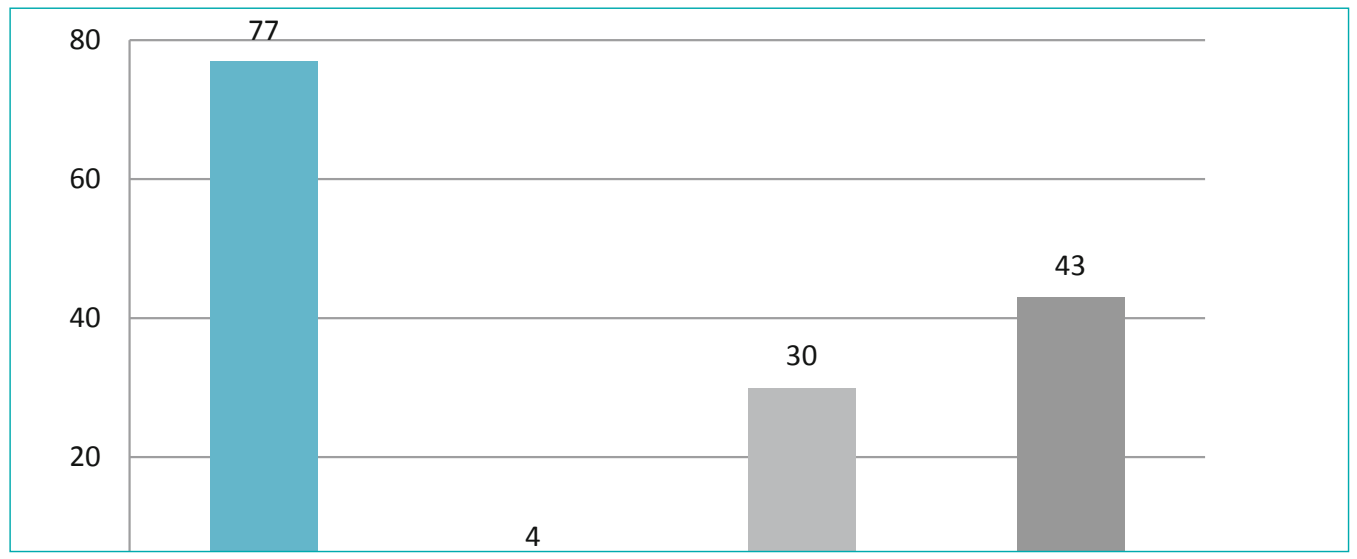

Abbildung 3: Fördermaßnahmen des Hypatia Programms von 2001 bis 2015, GD Gastdozentur, G-Prof Gastprofessur

Ein Hypatia Promotionsstipendium erhielten insgesamt 43 Absolventinnen der Beuth Hochschule.

\begin{tabular}{|llcc|}
\hline & Fachbereich der Beuth Hochschule & $\begin{array}{c}\text { geförderte } \\
\text { Absolven- } \\
\text { tinnen }\end{array}$ & $\begin{array}{l}\text { Erfolgreich } \\
\text { abgeschlossene } \\
\text { Promotionen }\end{array}$ \\
\hline I & Wirtschafts- und Gesellschaftswissenschaften & 2 & - \\
II & Mathematik - Physik - Chemie & 7 & 2 \\
III & Bauingenieur- und Geoinformationswesen & 5 & 2 \\
IV & Architektur und Gebäudetechnik & 5 & 1 \\
V & Life Sciences and Technology & 18 & 9 \\
VI & Informatik und Medien & 2 & 1 \\
VII & Elektrotechnik - Mechatronik -Optometrie & 1 & 1 \\
VIII $\quad$ Maschinenbau, Veranstaltungstechnik, Verfahrenstechnik & 3 & 3 \\
\hline & $\quad$ Gesamt & 43 & 19 \\
\hline
\end{tabular}

Tabelle 6: Hypatia Promotionsstipendiatinnen 2001 bis 2015 nach Fachbereichen

Mit 18 geförderten Promotionsvorhaben waren Absolventinnen des Fachbereichs V- Life Sciences and Technology - überproportional in der Hypatia Promotionsförderung vertreten, unter diesen waren 12 Absolventinnen des Studiengangs Biotechnologie. Dieser hohe Anteil der Biotechnologinnen an den durch das Hypatia Programm geförderten Promovendinnen, ist nicht zuletzt auf eine sehr hohe Motivation herausragender Absolventinnen des Studiengangs zur wissenschaftlichen Weiterqualifikation mit Blick auf die Verbesserung von Berufseinstiegsund Karrierechancen zurückzuführen.

Insgesamt 30 Gastdozenturen und vier Gastprofessoren wurden aus Hypatia Mitteln finanziert. Die als Gastdozentin und Gastprofessorinnen geförderten Nachwuchswissenschaftlerinnen waren durchweg in die Lehre und Forschung der Fachbereiche der Beuth Hochschule eingebunden, denen die Gastdozenturen zugeordnet waren. Zwei der Gastprofessorinnen wurden speziell für die 2009 bzw. 2010 neu eingerichteten Lehr- und Forschungsgebiete „Gender und Diversity“ und „Chancengerechtigkeit und Medienforschung“ ausgewählt. Diese neu einge-

${ }^{30}$ An zwei der geförderten Frauen wurde sowohl ein Promotionsstipendium als auch eine Gastdozentur vergeben. 
richteten Lehr- und Forschungsgebiete wurden in den Fachbereich I - Wirtschafts- und Gesellschaftswissenschaften - eingegliedert.

\begin{tabular}{|l|cc|}
\hline Fachbereich der Beuth Hochschule & $\begin{array}{l}\text { Gast- } \\
\text { dozentinnen }\end{array}$ & $\begin{array}{l}\text { Gast- } \\
\text { professorinnen }\end{array}$ \\
\hline I $\quad$ Wirtschafts- und Gesellschaftswissenschaften & 4 & 2 \\
II $\quad$ Mathematik - Physik - Chemie & 2 & - \\
III $\quad$ Bauingenieur- und Geoinformationswesen & 2 & - \\
IV Architektur und Gebäudetechnik & 9 & 1 \\
V $\quad$ Life Sciences and Technology & 5 & 1 \\
VI Informatik und Medien & 3 & - \\
VII Elektrotechnik - Mechatronik -Optometrie & - & - \\
VIII $\quad$ Maschinenbau, Veranstaltungstechnik, Verfahrenstechnik & 5 & - \\
\hline$\quad$ Gesamt & 30 & 4 \\
\hline
\end{tabular}

Tabelle 7:Hypatia Gastdozentinnen und Gastprofessorinnen 2001 bis 2015 nach Fachbereichen

\section{B1.2.3 Personengebundene Förderung des Hypatia Programms}

Eine Betrachtung der Förderverläufe und weiteren Berufsverläufe der in das Hypatia Programm aufgenommenen Absolventinnen und Nachwuchswissenschaftlerinnen der Hochschule auf Grundlage der statistischen bzw. qualitativen Erhebung zeigt, dass die Förderung zur Promotion bzw. zur Professur vor allem dann erfolgreich ist, wenn die nachstehenden vier Kriterien zusammenkommen und sich synergetisch ergänzen:

- Erstens, die geförderten Absolventinnen oder Nachwuchswissenschaftlerinnen der Hochschule ein wissenschaftliches fachbezogenes Erkenntnisinteresse haben.

- Zweitens, in einem Fachgebiet eine wissenschaftliche Weiterqualifikation - z.B. nachgewiesen durch die Promotion - die Grundvoraussetzung für eine Übernahme von Aufgaben in Lehre und Forschung ist.

- Drittens, die finanzielle fachbezogene Maßnahme als Kernelement einer Förderung in begleitende Maßnahmen eingebettet ist, wie ein fachbezogenes Begleitprogramm, ein überfachliches Qualifizierungsangebot, ein qualifiziertes Mentoring-/Betreuungsangebot von Beuth Lehrenden und ein individuales Coaching Angebot.

- Viertens, die Geförderten - auch die Promovendinnen - in die Strukturen und Einrichtungen der Beuth Hochschule eingebunden sind.

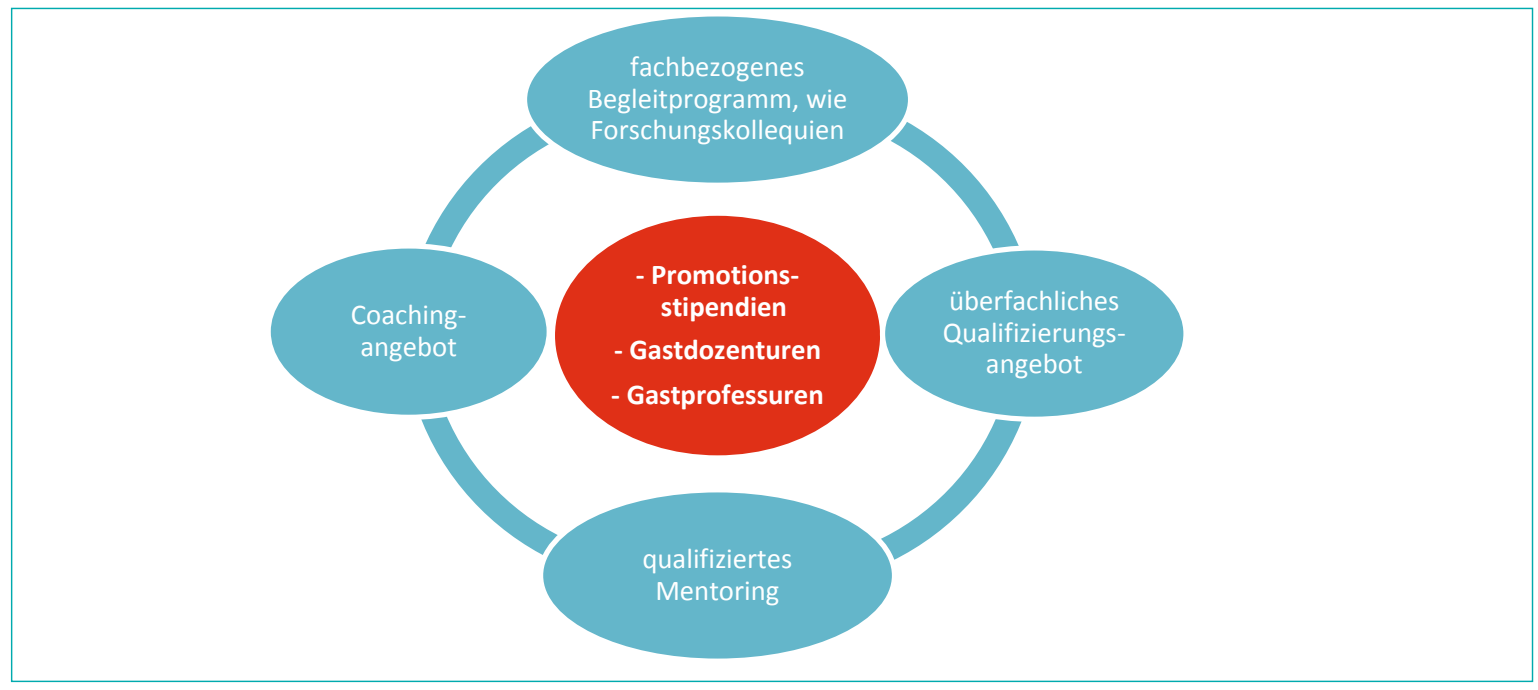

Abbildung 4: Aufbau des Hypatia Programms 
Diese für das Hypatia Programm der Beuth Hochschule ermittelten Eckpunkte einer erfolgreichen Förderung von weiblichen Wissenschaftskarrieren werden gestützt durch die Ergebnisse des Projekts „Fachkulturen und weibliche Wissenschaftskarrieren: Erfolgsfaktoren von Frauenförderung sichtbar gemacht“, das von 3/2011 bis 8/2013 vom Centrum für Hochschulentwicklung CHE durchgeführt wurde. (CHE, De Ridder et.al. 2013)

\section{B1.2.4 Erfolge - Promotionsförderung}

In dem Zeitraum von 2001 bis 12/2015 schlossen etwa die Hälfte

(19 Promovendinnen) der 39 Hypatia-Promotionsstipendiatinnen ihr Promotionsvorhaben erfolgreich ab, die vor 2014 in die Förderung aufgenommen wurden.

Vier weitere Beuth-Absolventinnen wurden erst 2014 in die Förderung aufgenommen und konnten durch das Hypatia Programm nicht mehr voll ausfinanziert werden, da Mittel für Promotionsstipendien des Hypatia Programms vom BCP ab 2016 nicht mehr bewilligt werden. Zwei der Absolventinnen, denen 2014 ein Hypatia Promotionsstipendium bewilligt wurde, konnten in das Promotionsprogramm der Beuth Hochschule übernommen werden ${ }^{31}$ und damit ihr begonnenes Promotionshaben in Anbindung an die Hochschule fortsetzen.

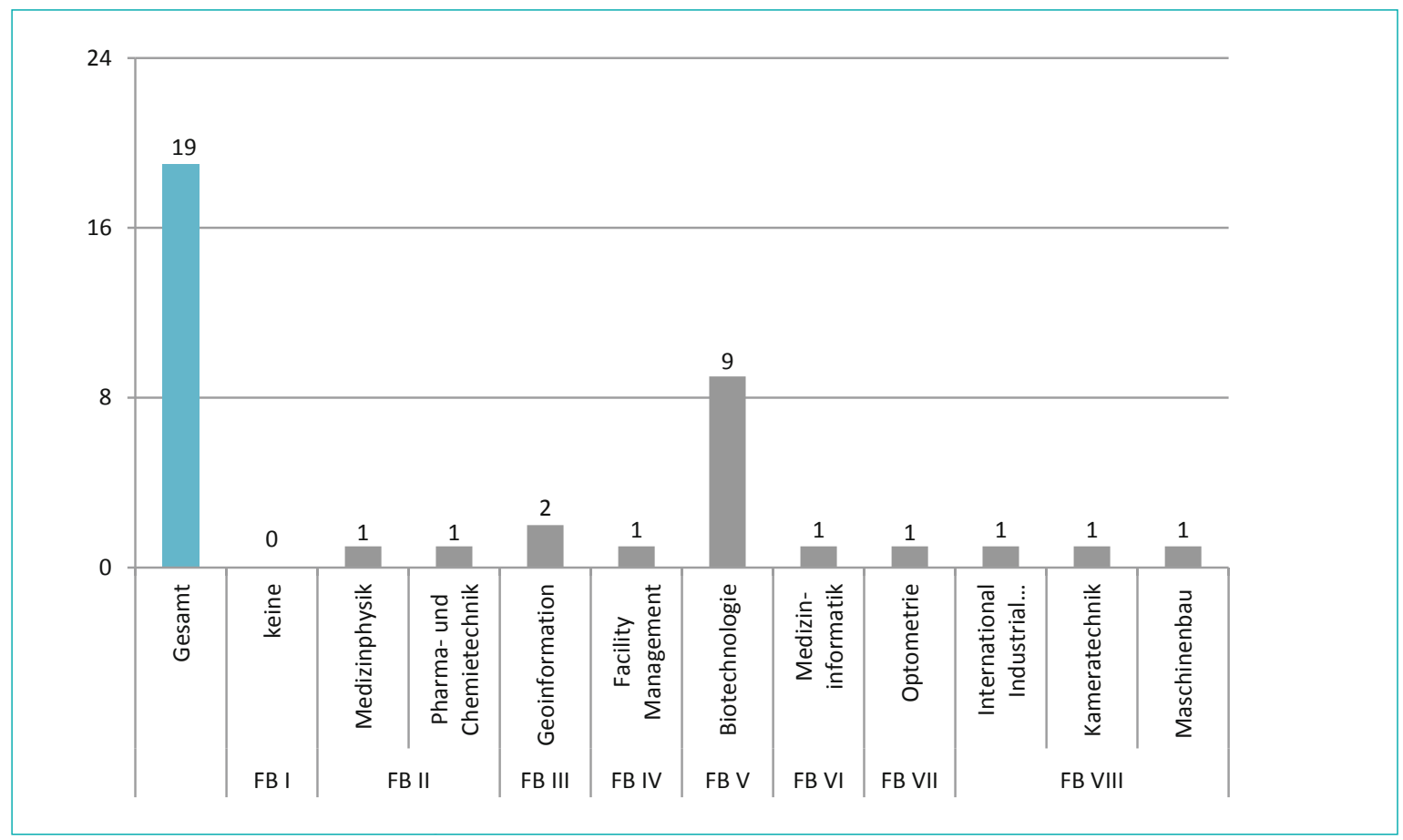

Abbildung 5: Anzahl der bis 12/2015 abgeschlossenen Promotionen

Die Hypatia Promovendinnen, die bis Ende 2015 ihre Promotion erfolgreich beendeten, waren überwiegend Absolventinnen von Studiengängen, die eine inhaltliche Affinität zu Themenbereichen, Methoden und Fragen der angewandten Wissenschaft und Forschung haben, wie Biotechnologie (9 von 12 Geförderten), Pharma- und Chemietechnik (1 von 4 Geförderten), Medizinphysik (1 von 3 Geförderten), Medizininformatik (1 von 1 Geförderten) oder Geoinformation (2 von 4 Geförderten). Aus diesen fünf Studiengängen wurden 25 Absolventinnen gefördert, 14 schlossen das Promotionsverfahren erfolgreich ab.

Hypatia Promotionsstipendiatinnen, die ein Studium eines Studiengangs mit einer inhaltlichen Affinität zu Gestaltung, Kunst, Ästhetik oder Vermittlung von Wissen zur Lösung von konkreten Praxis- und Forschungsfragen absolviert haben, schließen ihre Promotion seltener ab. Dieser Fächergruppe können die Studiengänge Druck- und Medientechnik (1 Geförderte), Bauingenieurwesen (1 Geförderte), Architektur (4 Geförderte), Landschaftsplanung (1 Geförderte), Landschaftsarchitektur (2 Geförderte), Gartenbau (1 Geförderte), Urbanes Pflanzen- und Freiraum-Management (1 Geförderte) sowie Verpackungstechnik (1 Geförderte) zugeordnet

${ }^{31}$ vgl. S. 43 
werden. Von den 12 Absolventinnen dieser Studiengänge, die im Förderzeitraum von 2001 bis 2014 ein Hypatia Stipendium erhielten, war bis Ende 12/2015 von keiner Geförderten bekannt, dass die Promotion erfolgreich abgeschlossen wurde.

In dieser Fächergruppe kommt hinzu, dass eine Promotion nicht unabdingbar die formale Voraussetzung für eine Karriere in der Lehre und Forschung ist und auch für eine Berufskarriere in der Praxis eher weniger Bedeutung hat.

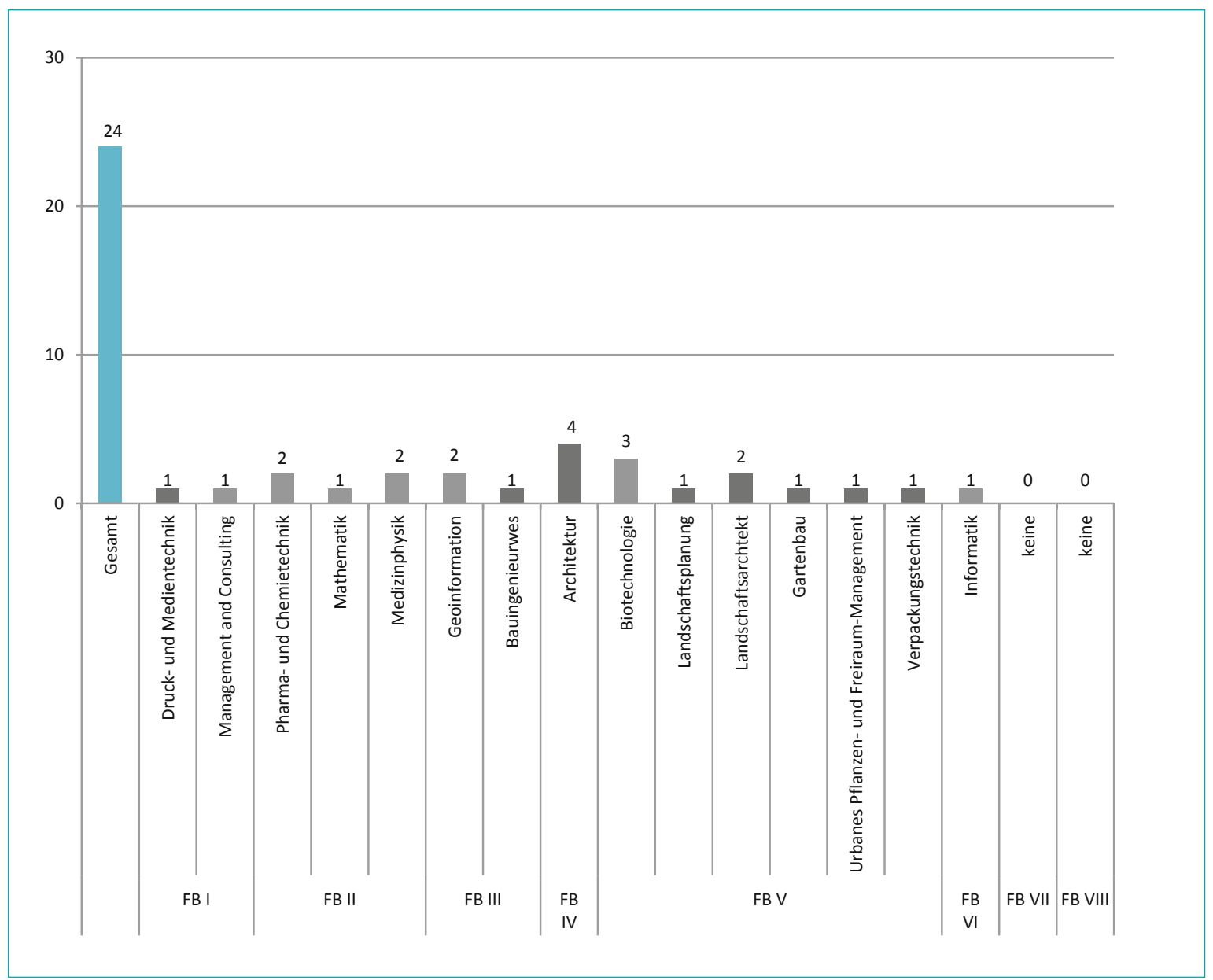

Abbildung 6: Anzahl der bis 12/2015 nicht abgeschlossenen Promotionen

Doch Studiengänge bzw. Studieninhalte sind nur ein erster Hinweis darauf, ob eine Promotionsförderung erfolgreich oder weniger erfolgreich verläuft.

Beim Blick auf die abgeschlossenen Promotionen der Hypatia geförderten Frauen fällt des Weiteren auf, dass die Professorinnen und Professoren der Beuth Hochschule, die als Mentoren oder Mentorinnen eine promovierende Absolventin der Hochschule begleiteten, maßgeblich den Verlauf eines Promotionsvorhabens mit beeinflussten. Die wissenschaftliche und wissenschaftspolitische Positionierung und Vernetzung der Mentoren und Mentorinnen der Beuth Hochschule sowie deren Ausgestaltung der wissenschaftlichen Betreuung sind - zumindest für FH-Absolventinnen der Beuth Hochschule - für einen erfolgreichen Promotionsabschluss und auch anschließenden schnellen Übergang der Geförderten auf eine Professur wegweisend oder bedeutend für einen sehr guten Berufseinstieg nach der Promotion.

Hier einige Beispiele: ${ }^{32}$

Zwei der Hypatia Stipendiatinnen wurden sehr schnell nach Abschluss ihrer Promotion auf Professuren berufen:

- Prof. Dr. A, Promotion 2003, 2004 erste Professur, heute Präsidentin einer Fachhochschule.

- Prof. Dr. B, Promotion 2003, 2008 Berufung auf eine Professur an eine Hochschule.

${ }^{32}$ Für diese Veröffentlichung wurden alle Namen anonymisiert. Die Personen sind den Autorinnen bekannt. 
Diese beiden erfolgreichen Wissenschaftlerinnen hatten einen Mentor bzw. eine Mentorin, der bzw. die dem Leitungsgremium der Beuth Hochschule angehörten.

Neben Mitgliedern des Leitungsgremiums der Beuth Hochschule sind es vor allem Dekane und Dekaninnen, die „ihre“ Hypatia Promovendinnen zum erfolgreichen Promotionsabschluss begleiteten. Dies trifft u. a. zu für:

- Fachbereich III - Bauingenieur- und Geoinformationswesen:

Frau Dr. C und Frau Dr. D. Die beiden Hypatia Stipendiatinnen schlossen ihre Promotion 2015 erfolgreich ab. Frau Dr. C arbeitet inzwischen entsprechend ihrer Qualifikation in einem Landesbetrieb. Frau Dr. D baut in einem ostafrikanischen Land eine Forschungskooperation mit der Beuth Hochschule im Rahmen eines Projekts zur Entwicklung eines geographischen Informationssystems zur Malaria auf.

- Fachbereich IV - Architektur und Gebäudetechnik

Frau Dr. E, Promotion 2015, übernahm nach dem Promotionsabschluss Aufgaben in Lehre und Forschung als Lehrbeauftragte an einer Hochschule und wechselte sehr schnell in das Management eines großen weltweiten Unternehmens.

- Fachbereich VIII - Maschinenbau, Veranstaltungstechnik, Verfahrenstechnik

Frau Dr. F, bisher einzige erfolgreiche Hypatia Promovendin mit Erstabschluss Maschinenbau, Promotion 2013 - ihre Mentorin gehörte dem Leitungsgremium der Beuth Hochschule an und ist heute Präsidentin einer Hochschule.

- Ein Mentor des Fachbereichs VIII hat seinen eigenen Weg in die Wissenschaft quasi als Blaupause für die Betreuung seiner Mentee, Frau Dr. G, gewählt. Frau Dr. G ist heute eine erfolgreiche mehrfach ausgezeichnete Regisseurin und Drehbuchautorin.

Professorinnen und Professoren, die es sich zu Aufgabe gemacht haben, die wissenschaftliche Weiterqualifikation ihre besonders herausragenden Studentinnen zu fördern, sind ein weiterer Garant für erfolgreiche Promotionsverläufe und Wege in die Forschung. Die neun erfolgreichen Promovendinnen der Biotechnologie verteilen sich zum Beispiel auf zwei Professoren (erfolgreiche Promotionen 3 und 2) und zwei Professorinnen (erfolgreiche Promotionen 3 und 1) des Studiengangs, die kontinuierlich engagiert Absolventinnen ihres Studiengangs betreut haben bzw. noch betreuen.

Als ein Resümee ist noch einmal festzuhalten, dass ein tragendes Fundament für eine erfolgreiche Promotion und für einen erfolgreichen Berufseinstieg gegeben ist, wenn wissenschaftliches Erkenntnisinteresse der Promovendinnen, eine Affinität der Studieninhalte zu Themenbereichen, Methoden und Fragen der angewandten Wissenschaft und Forschung sowie eine intensive wissenschaftliche Betreuung und wissenschaftspolitisches Engagement der Mentoren bzw. Mentorinnen mit Anbindung an die Beuth Hochschule aufeinander treffen.

Das zeigt auch der Blick auf den weiteren Berufsweg der Hypatia Stipendiatinnen, die ihre Promotion bis Ende 2015 erfolgreich abgeschlossen hatten. Neben den zwei bereits genannten Professorinnen haben 11 der ehemaligen Hypatia Stipendiatinnen Aufgaben in der Forschung und drei Managementaufgaben übernommen. Eine arbeitet - wie ebenfalls vorstehend bereits ausgeführt - als Regisseurin. Nur von zwei der 19 Hypatia Stipendiatinnen, die ihre Promotion erfolgreich abgeschlossen haben, war Ende 2015 über ihren Tätigkeitsbereich nichts bekannt.

\section{B1.2.5 Erfolge - Gastdozenturen}

Von 2001 bis 2015 wurden vom Hypatia Programm 30 Gastdozenturen bewilligt. Insgesamt erhielten sieben Absolventinnen der TFH bzw. Beuth Hochschule, sieben Absolventinnen anderer Fachhochschulen bzw. Akademien und 16 Absolventinnen von Universitäten eine Hypatia Gastdozentur.

Nur acht der 30 Nachwuchswissenschaftlerinnen waren bei Antritt der Gastdozentur promoviert, zwei Absolventinnen der TFH bzw. Beuth Hochschule und sechs Absolventinnen von Universitäten. 
Fünf der Nachwuchswissenschaftlerinnen - eine TFH bzw. Beuth Absolventin und vier Universitätsabsolventinnen - schlossen ihr Promotionsvorhaben nach der Gastdozentur erfolgreich ab. Sowohl eine Beuth als auch eine Universitäts-Absolventin arbeiteten Ende 2015 noch an ihrem Promotionsvorhaben. Die Hälfte (15) der 30 Hypatia Gastdozentinnen des Zeitraums 2001 bis 2015 waren Ende 2015 nach wie vor nicht promoviert und es ist auch nicht bekannt, dass sie eine Promotion anstreben. In diesem letztgenannten Sample sind die sieben Gastdozentinnen des Bereichs Architektur, die bei Antritt der Gastdozentur und auch Ende 2015 nicht promoviert waren.

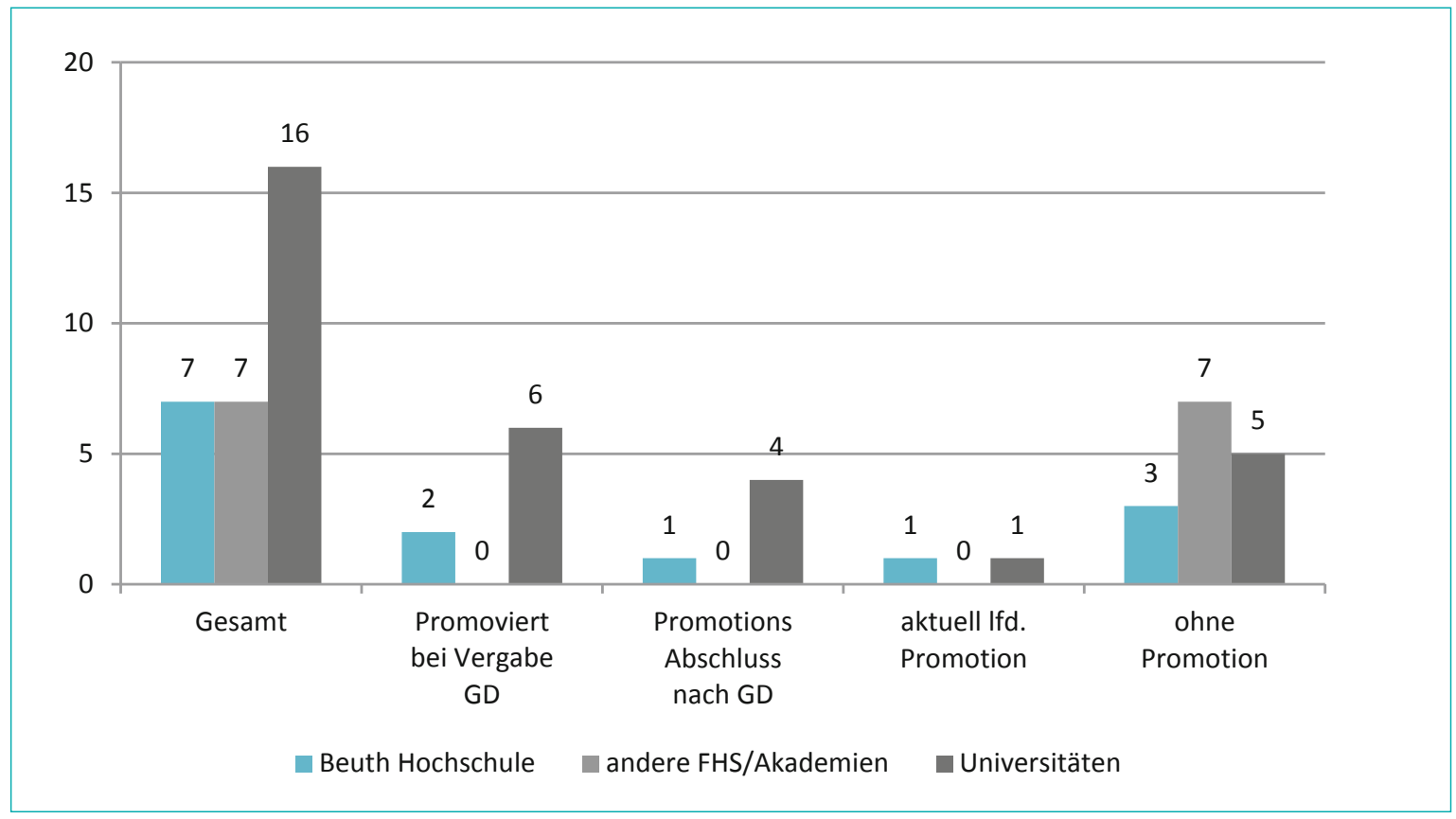

Abbildung 7: Hypatia Gastdozentinnen 2001 bis 2015 - Promotionen nach Herkunftshochschule

Erfreulich ist, dass von den 30 Hypatia Gastdozentinnen fünf bis 12/2015 auf eine Professur berufen wurden, zwei von ihnen auf eine Professur an der Beuth Hochschule. Dies sind:

- Frau Prof. Dr. GD1, bis 2015 Dekanin ihres Fachbereichs. Sie ist Absolventin der Beuth Hochschule und begann ihre Wissenschaftskarriere als Hypatia Promotionsstipendiatin.

- Frau Prof. Dr. GD2 promovierte nach Abschluss der Gastdozentur im Rahmen ihrer Berufstätigkeit erfolgreich und erhielt an der Beuth Hochschule einen Ruf auf ihre derzeitige Professur.

Durchweg blieben die Nachwuchswissenschaftlerinnen nach Ablauf der Hypatia Gastdozentur eng mit der Beuth Hochschule vernetzt und übernahmen als Lehrbeauftragte oftmals für mehrere Semester an der Hochschule Aufgaben in der Lehre, bevor sie auf eine Professur berufen wurden, als Mitarbeiterinnen eine Tätigkeit in der Forschung übernahmen oder sich eine Selbstständigkeit aufgebaut hatten.

\section{B1.2.6 Erfolge der Gastprofessorinnen}

2009 und 2010 konnte das GuTZ in Kooperation mit der zentralen Frauenbeauftragten aus dem quotierten Bereich des BCP erfolgreich Mittel zur Einrichtung von Gastprofessuren für die neuen Fachgebiete Digitalisierung und Chancengerechtigkeit sowie Gender und Diversity im Studium Generale des Fachbereichs I - Wirtschafts- und Gesellschaftswissenschaften der Beuth Hochschule einwerben.

- 2011 übernahm Frau Prof. Dr. GP1 eine der zwei neu geschaffenen Gastprofessuren. Frau Prof. Dr. GP1 hat inzwischen den Ruf auf eine ordentliche Professur an der Beuth Hochschule erhalten, angenommen und angetreten.

- Die 2009 neu eingerichtete Gastprofessur wurde mit Frau Dr. GP2 besetzt. Frau Dr. GP2 gab die Gastprofessur vor Ablauf zurück, um für eine politische Stiftung die Leitung des amerikanischen Regionalbüros der Stiftung zu übernehmen. Für den dadurch zur Disposition ste- 
henden Aufgabenbereich der Gastprofessur erhielt Frau GD3 eine Hypatia Gastdozentur. Nach dem Auslaufen ihrer Gastdozentur übernahm Frau GD3 als Lehrbeauftragte Aufgaben in der Lehre und war Mitglied des GuTZ der Beuth Hochschule.

Zwei weitere Hypatia Gastprofessuren wurden vergeben an:

- Frau Dr. GP3. Als Gastprofessorin war sie dem Fachbereich IV - Architektur und Gebäudetechnik - zugeordnet. Zum Zeitpunkt der Übernahme der Gastprofessur war Frau Dr. GP3 noch im Promotionsverfahren, ihre Promotion schloss sie zu einem späteren Zeitpunkt ab.

- Frau Dr. GP4. Die Gastprofessur wurde an den Fachbereich V - Life Sciences and Technology vergeben. Nach ihrem TFH-Masterabschluss hatte Frau Dr. GP4 promoviert. Inzwischen ist sie habilitiert und lehrt als Lehrbeauftragte und Privatdozentin.

Festzuhalten ist, dass die beiden Gastprofessorinnen, die Aufgaben in innovativen Lehr- und Forschungsgebieten übernommen haben, ihre Berufskarriere besonders erfolgreich fortsetzen konnten: Eine schaffte den Sprung auf eine ordentliche Professur und die Zweite in die internationale Wissenschafts- und Forschungspolitik.

\section{B1.2.7 Zusammenstellung der Erfolge und Verbleib der Geförderten}

Als Ergebnis bleibt festzuhalten, dass von den von 2001 bis 2015 insgesamt 75 geförderten Wissenschaftlerinnen acht auf eine Professur berufen wurden.

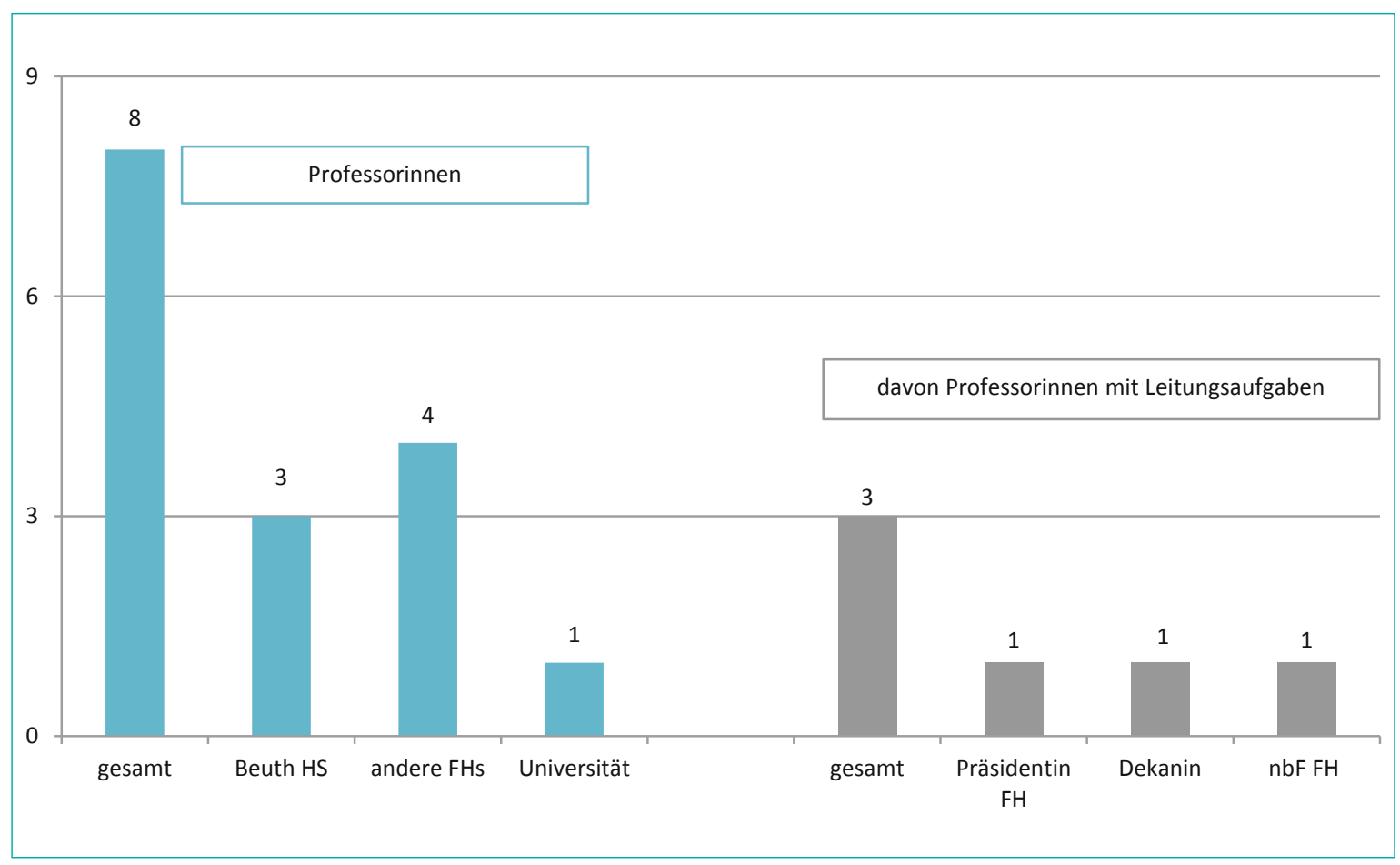

Abbildung 8: Hypatia Förderzeit 2001 bis 2015 - Professorinnen und Leitungspositionen

- Drei der acht berufenen Wissenschaftlerinnen erhielten Professuren an der Beuth Hochschule.

- Vier der acht Professorinnen lehren und forschen an anderen Fachhochschulen; eine von ihnen leitet inzwischen die Fachhochschule, an die sie berufen wurde.

- Eine der acht Wissenschaftlerinnen wurde nach Abschluss der Hypatia Gastdozentur direkt auf eine C4-Professur berufen.

- Von den acht Professorinnen haben drei ein Studium an der Beuth Hochschule absolviert. 


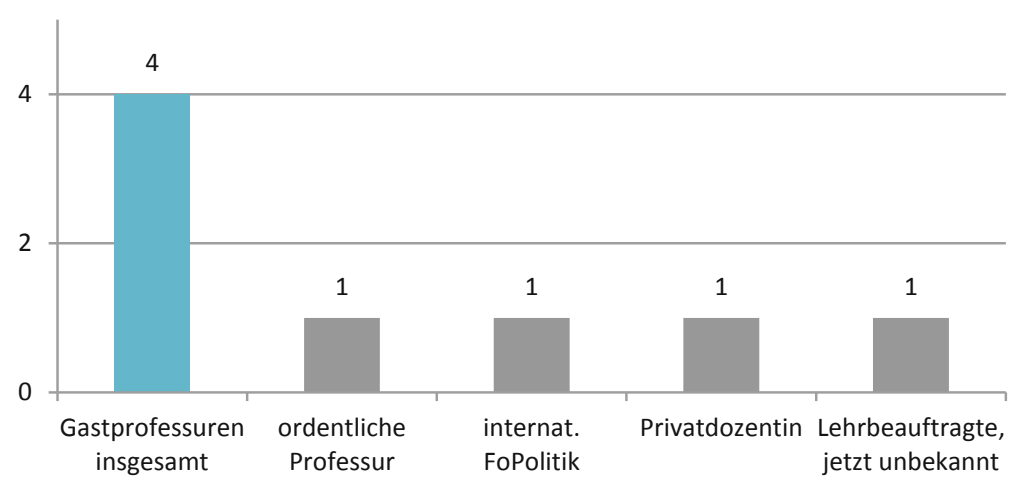

Abbildung 9: Verbleib Gastprofessorinnen - Stand 12/2015

Eine ehemalige Gastprofessorin leitet das US-amerikanische Auslandsbüro einer politischen Stiftung; eine der Gastprofessorinnen ist inzwischen habilitiert und als Privatdozentin an der habilitierenden Universität tätig.

Eine der Gastprofessorinnen war an der Beuth Hochschule weiterhin in die Lehre eingebunden, über ihre aktuelle Berufstätigkeit ist nichts bekannt.

\section{Hypatia Gastdozenturen}

Fünf der 30 Hypatia Gastdozentinnen wurden auf eine Professur berufen, zwei von ihnen auf eine Professur an der Beuth Hochschule.

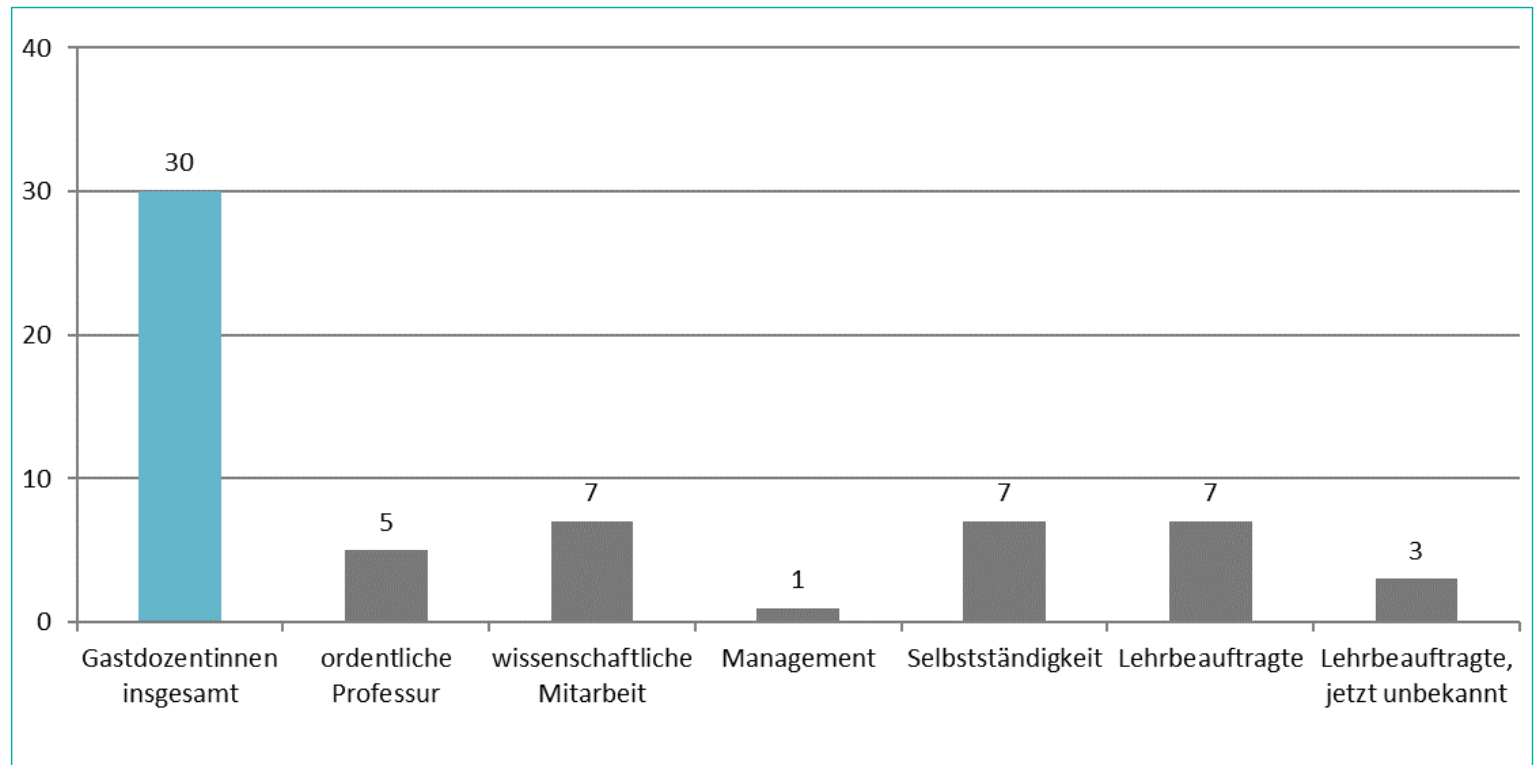

Abbildung 10: Verbleib Gastdozentinnen - Stand 12/2015

Ende 2015 arbeiteten sieben der ehemaligen Hypatia Gastdozentinnen als wissenschaftliche Mitarbeiterinnen, eine hatte Managementaufgaben übernommen, sieben weitere haben sich eine Selbstständigkeit aufgebaut und ebenfalls sieben waren als Lehrbeauftragte tätig. Von drei der ehemaligen Hypatia Gastdozentinnen konnte über ihren Verbleib nichts in Erfahrung gebracht werden. 


\section{Hypatia Promotionsstipendiatinnen}

Aus der Gruppe der 19 Hypatia Promotionsstipendiatinnen, die ihre Promotion erfolgreich abschlossen, erhielten zwei bis 12/2015 eine Professur.

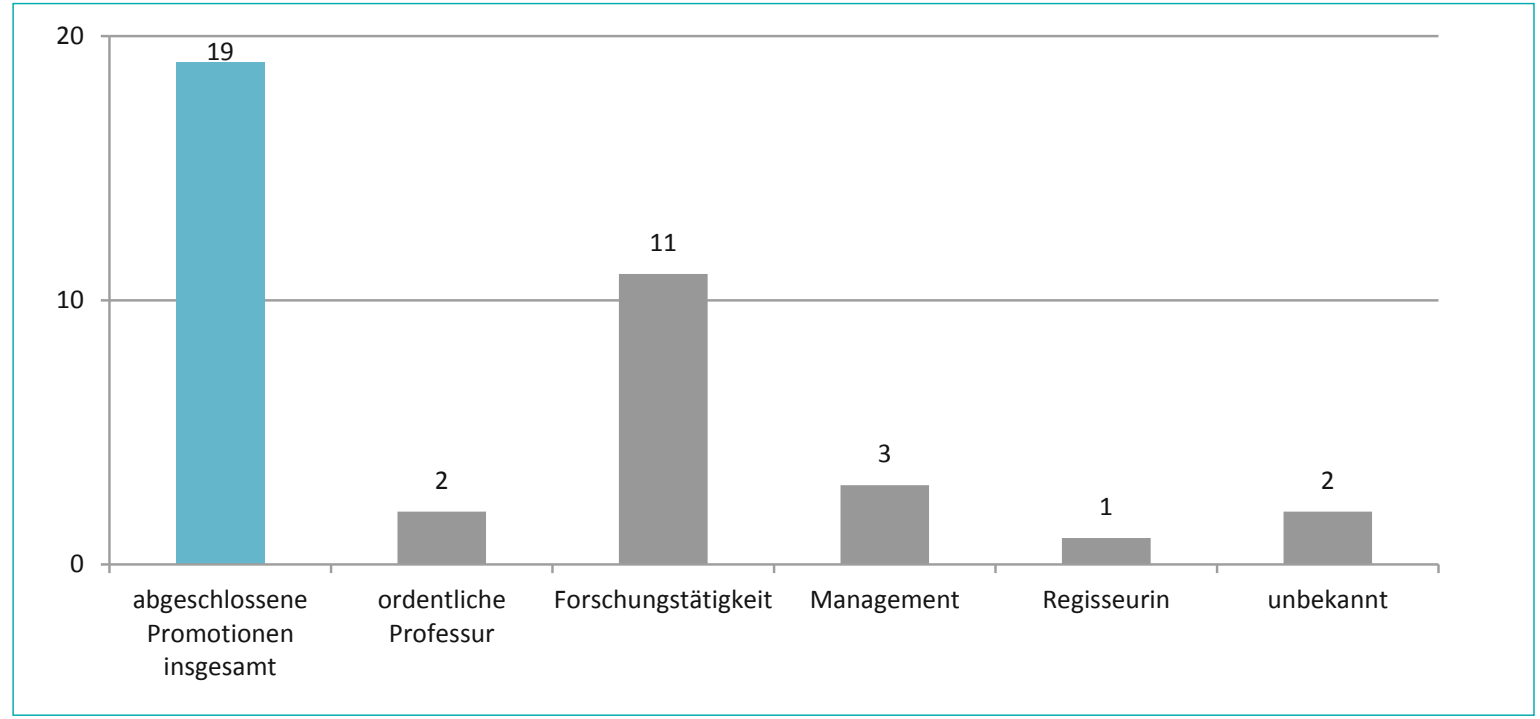

Abbildung 11: Verbleib erfolgreiche Promotionsstipendiatinnen - Stand 12/2015

Von sieben der neun erfolgreichen Hypatia Promotionsstipendiatinnen, die ein Studium der Biotechnologie abgeschlossen haben, ist bekannt, dass sie eine Forschungstätigkeit ausüben; der Berufsverlauf der zwei weiteren ist nicht bekannt.

Von den acht erfolgreichen Hypatia Promotionsstipendiatinnen mit anderen Studienabschlüssen arbeiten vier ebenfalls in Forschungseinrichtungen oder sind in Forschungskooperationen eingebunden, drei haben leitende Managementaufgaben übernommen und eine ist Regisseurin.

Insgesamt 24 Absolventinnen der Hochschule, die in die Hypatia Promotionsförderung aufgenommen wurden, schlossen ihre Promotion bis Ende 2015 nicht ab. Vier von ihnen hatten erstmals 2014 ein Hypatia Promotionsstipendium erhalten. Aufgrund der ab 2016 für Hypatia Stipendien nicht mehr vorhandenen Mittel mussten die Promovierenden sich nach anderen Fördermöglichkeiten umsehen. Zwei bewarben sich erfolgreich auf Stipendien, die von der Forschungsabteilung der Beuth Hochschule für Promotionen vergeben werden.

Von den 20 weiteren übernahmen fünf nach dem Auslaufen der Promotionsförderung wissenschaftliche Tätigkeiten in Einrichtungen, Instituten oder Unternehmen; eine ist nach wie vor als Lehrbeauftragte an der Beuth Hochschule angebunden, sechs arbeiten als Mitarbeiterinnen entsprechend ihrem Studienabschluss an, drei orientierten sich um und bauten sich eine Selbstständigkeit auf - eine Architektin und zwei Landschaftsarchitektinnen. Der weitere Berufsverlauf konnte für fünf ehemalige Hypatia Promotionsstipendiatinnen, die bis Ende 2015 ihr Promotionsvorhaben nicht abgeschlossen hatten, nicht ermittelt werden. 


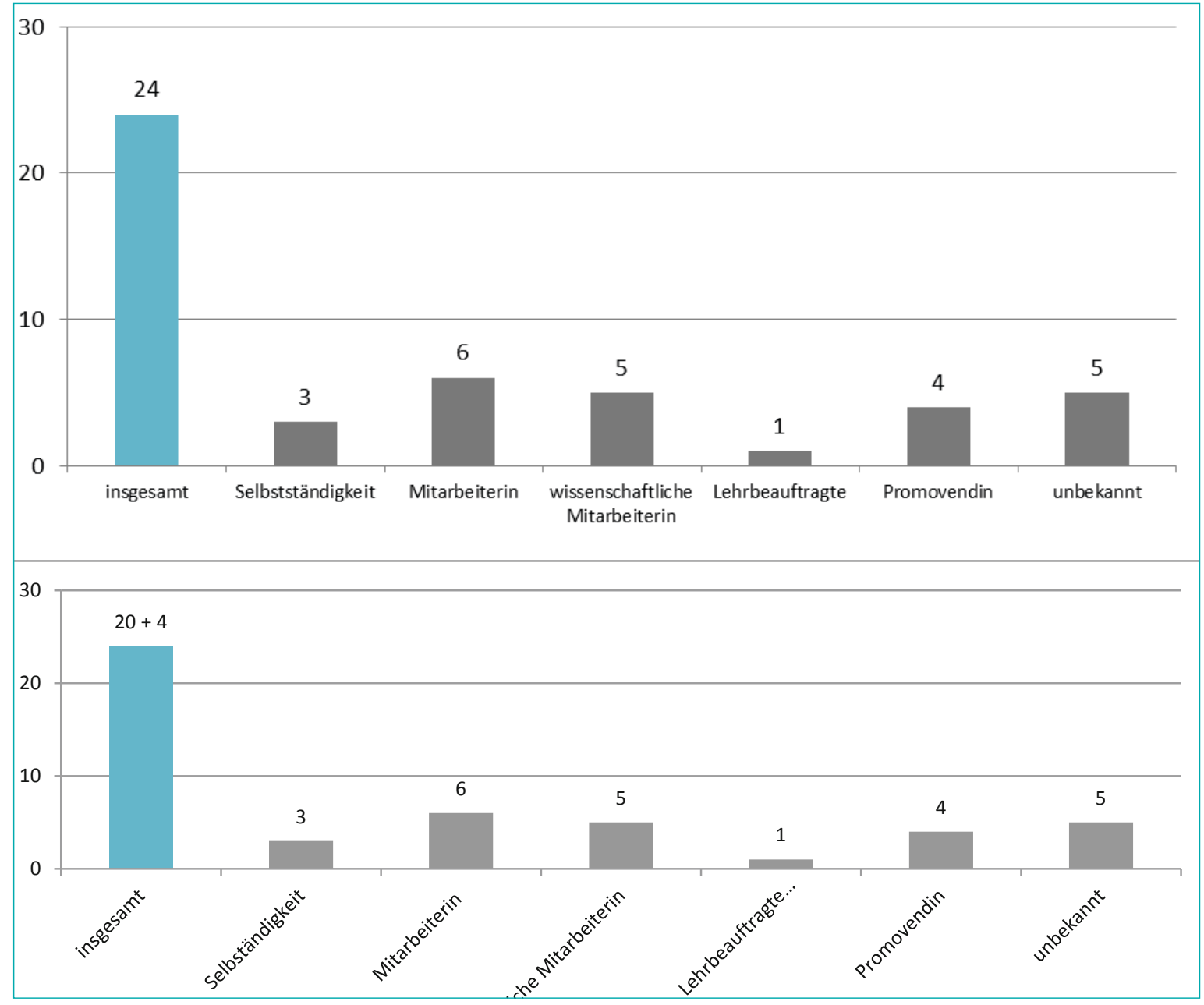

Abbildung 12: Verbleib Hypatia Stipendiatinnen ohne Promotionsabschluss bis 12/2015

38

Diallo-Ruschhaupt, Plaumann \& Dombrowski: Karrierewege zur Professur 


\section{B2 Wege von Beuth Absolvierenden zur Promotion - Szenario eines Hindernislaufs}

20 Jahre nach dem Beschluss zur Öffnung der Universitäten für Promotionen von besonders qualifizierten Fachhochschul-Absolvierenden wäre es zu weit gegriffen davon zu sprechen, dass Promotion und Wissenschaftskarriere bis zur Professur für Absolventinnen der Beuth Hochschule keine Ausnahme mehr, sondern zur Alltäglichkeit geworden ist.

Denn, Absolvierende mit einem FH-Abschluss müssen - anders als ihre Kommilitonen oder Kommilitoninnen mit Universitätsabschluss - vielfältige zusätzliche Organisationsleistungen erbringen sowie besondere universitätsinterne Entscheidungsprozesse bezüglich der Anerkennung und des Nachweises ihrer Befähigung zum wissenschaftlichen Arbeiten durchlaufen, bevor sie wie alle Promovierenden den mit Stolpersteinen gepflasterten Weg der Bearbeitung eines Forschungsthemas bis zu erfolgreichen Promotion beschreiten können.

Erst einmal müssen FH-Absolvierende an einer Universität - einer Einrichtung, deren Strukturen und Lehrende ihnen nicht bekannt sind -, einen Professor oder eine Professorin finden, der bzw. die ihr Promotionsvorhaben unterstützt.

Deshalb gehörte es zum Konzept des Hypatia Programms, dass Absolvierende der Beuth Hochschule mit einem Beuth-Professor oder eine Beuth-Professorinnen „ihres Vertrauens“ ihre Promotionsidee ohne Erfolgsdruck vorbesprechen und/oder von dem Professor oder der Professorin ermutigt, motiviert und unterstützt werden, ein Promotionsvorhaben zu planen.

\begin{tabular}{|c|c|c|c|c|c|c|}
\hline & \multicolumn{6}{|c|}{ Vorbereitung der Promotion } \\
\hline & Promotionsidee & $\begin{array}{l}\text { Mit Professor oder } \\
\text { Professorin des } \\
\text { Vertrauens der } \\
\text { Beuth Hochschule } \\
\text { Vorgespräch zur } \\
\text { Promotionsidee }\end{array}$ & $\begin{array}{l}\text { Welche Universi- } \\
\text { tät passt und wie } \\
\text { sind dort die } \\
\text { Zulassungsbe- } \\
\text { dingungen zur } \\
\text { Promotion für FH- } \\
\text { absolvierende } \\
\end{array}$ & $\begin{array}{l}\text { Auswahl und } \\
\text { Vorgespräch mit } \\
\text { dem möglichen } \\
\text { Erstgutachter bzw. } \\
\text { der möglichen } \\
\text { Erstgutachterin }\end{array}$ & $\begin{array}{l}\text { Auswahl promo- } \\
\text { vierende Universi- } \\
\text { tät und Erstgut- } \\
\text { achter bzw. } \\
\text { Erstgutachterin } \\
\text { erfolgreich } \\
\text { abgeschlossen } \\
\end{array}$ & \begin{tabular}{|l|} 
Erstellung des \\
Exposés zur \\
Promotion in \\
Abstimmung mit \\
dem Erstgutachter \\
bzw. der Erstgut- \\
achterin \\
\end{tabular} \\
\hline & \multicolumn{6}{|c|}{ Verfahren auf Zulassungzur Promotion und Finanzierung der Promotion } \\
\hline & \multirow{2}{*}{$\begin{array}{l}\text { Einreichung des } \\
\text { Antrags auf } \\
\text { Zulassung zur } \\
\text { Promotion an der } \\
\text { promovierenden } \\
\text { Universität durch } \\
\text { den Erstgutachter } \\
\text { bzw. die Erstgut- } \\
\text { achterin }\end{array}$} & $\begin{array}{l}\text { Entscheidung des } \\
\text { zuständigen } \\
\text { Universitäts- } \\
\text { gremiums: } \\
\text { Annahme zur } \\
\text { Promotion }\end{array}$ & \multirow[t]{2}{*}{$\begin{array}{l}\text { Erfüllung der } \\
\text { Auflagen durch } \\
\text { den FH- } \\
\text { Absolventen bzw. } \\
\text { die FH-Absolventin }\end{array}$} & \multirow{2}{*}{$\begin{array}{l}\text { Akquise zu } \\
\text { Finanzierungs- } \\
\text { möglichkeiten der } \\
\text { Promotionsphase, } \\
\text { Rücksprache mit } \\
\text { Mentor bzw. } \\
\text { Mentorin der } \\
\text { Beuth Hochschule }\end{array}$} & \multirow{2}{*}{ 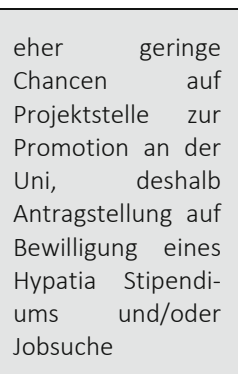 } & $\begin{array}{l}\text { Hypatia Stipendi- } \\
\text { um bewilligt } \\
\text { und/oder Job } \\
\text { gefunden }\end{array}$ \\
\hline & & $\begin{array}{l}\text { ggf. mit Auflagen } \\
\text { zur Belegung } \\
\text { bestimmter } \\
\text { Veranstaltungen } \\
\text { mit Prüfung }\end{array}$ & & & & $\begin{array}{l}\text { Finanzierung der } \\
\text { Promotionsphase } \\
\text { für } 2 \text { ggf. } 3 \text { Jahre } \\
\text { gesichert }\end{array}$ \\
\hline & \multicolumn{6}{|c|}{ Promotionsphase } \\
\hline & $\begin{array}{l}\text { Im Hypatia } \\
\text { Programm bereits } \\
\text { angeboten }\end{array}$ & \multicolumn{5}{|c|}{ Mentoring durch den ausgewählten Professor bzw. die ausgewählte Professorin der Beuth Hochschule } \\
\hline$\downarrow$ & $\begin{array}{l}\text { Bearbeitung des } \\
\text { Promotions- } \\
\text { themas }\end{array}$ & $\begin{array}{l}\text { qualifizierte } \\
\text { fachliche Betreu- } \\
\text { ung }\end{array}$ & $\begin{array}{l}\text { Vermittlung von } \\
\text { internationalen } \\
\text { Kontakten }\end{array}$ & $\begin{array}{l}\text { Vorbereitung zur } \\
\text { Übernahme von } \\
\text { Lehrtätigen - } \\
\text { Vermittlung von } \\
\text { Lehraufträgen }\end{array}$ & $\begin{array}{l}\text { Einbindung in } \\
\text { Wissenschafts- } \\
\text { und Forschungs- } \\
\text { netzwerke der } \\
\text { Profession und der } \\
\text { Beuth Hochschule }\end{array}$ & $\begin{array}{l}\text { Übernahme von } \\
\text { Zweit- oder } \\
\text { Drittbegut- } \\
\text { achtungsaufgaben }\end{array}$ \\
\hline & $\begin{array}{l}\text { Im Hypatia } \\
\text { Programm } \\
\text { zeitweise bereits } \\
\text { angeboten }\end{array}$ & \multicolumn{5}{|c|}{ Internationales fachliches und überfachliches Begleitprogramm } \\
\hline & \multicolumn{6}{|c|}{ Erfolgreicher Abschluss der Promotion } \\
\hline
\end{tabular}

Abbildung 13: Hypatia Promotionsstipendiatinnen Förderung und Begleitung an der TFH bzw. Beuth Hochschule 2001 bis 2015

Wünschenswert war des Weiteren, dass der jeweilige Professor oder die jeweilige Professorin der Beuth Hochschule dem bzw. der Promotionswilligen Empfehlungen für eine Universität und für einen Doktorvater bzw. für eine Doktormutter unterbreiteten sowie vermittelnd den 
Prozess des Kennenlernens von zukünftigem Doktorvater bzw. zukünftiger Doktormutter und dessen zukünftigen Doktoranden bzw. zukünftiger Doktorandin aktiv begleiteten. Denn Leistungen und Habitus des Beuth Absolvierenden bzw. der Beuth Absolvierenden mit Promotionsabsicht sind dem ausgewählten Beuth Professor bzw. der ausgewählten Beuth Professorin zumeist aus dem Studium gut bekannt und dem zukünftigen Doktorvater bzw. der zukünftigen Doktormutter am besten nahegebracht werden.

Wenn dieses erste Hindernis mit Bravour genommen wurde und auch der Antrag auf Annahme als Doktorand bzw. Doktorandin vollständig und qualifiziert dem zuständigen Universitätsgremium zur Entscheidung vorliegt, tritt vielfach schon das nächste Hindernis in den Blickpunkt.

Auch mit einem herausragenden Masterabschluss ist es nicht selbstverständlich, dass eine Zulassung zur Promotion an einer Universität ohne Auflagen erfolgt. Universitäts- und Masterabschlüsse werden mittlerweile zwar vielfältig als gleichwertig angesehen, aber nicht immer. Oftmals wird von einer promovierenden Universität die Zulassung zur Promotion noch mit Auflagen ausgesprochen, wie eine erfolgreiche Teilnahme an festgesetzten Seminaren, Vorlesungen oder Prüfungen.

Die Erfüllung der Auflagen kostet den promotionswilligen FH-Absolvierenden zusätzliche Zeit. Dies geht zu Lasten einer zügigen Bearbeitung des Promotionsvorhabens, d.h. der Zeitplan zur Durchführung des Promotionsvorhabens muss entsprechend angepasst werden. Damit erhöht sich auch der Mittelbedarf zur Ausfinanzierung des Promotionsvorhabens.

Das Hypatia Programm hatte bis Ende 2009 durch entsprechende Vergaberichtlinien für die Stipendien, den zusätzlichen Zeitbedarf zur Erfüllung der universitären Auflagen für promotionsmotivierte Absolventinnen der Hochschule durch die Bewilligung von Vorbereitungsstipendien abgefedert. Mit dem Auslaufen der Diplom-Studiengänge wurden vom BCP ab 2010 Mittel für Vorbereitungsstipendien grundsätzlich nicht mehr genehmigt. Die FrauenFörderKommission passte entsprechend die formalen Vergabekriterien des Hypatia Programms an und vergab nach sorgfältiger Prüfung 6-monatige Stipendien zur Vorbereitung einer Promotion ab 2010 nur noch in begründeten Ausnahmefällen bei einer gravierenden Nicht-Anerkennung der Studienleistungen einer Masterabsolventin durch eine Universität. ${ }^{33}$

Ein weiteres Kriterium im Prozess der Vorbereitung einer Promotion ist die Finanzierung des Lebensunterhalts während des Promotionszeitraums.

Für alle Promotionswilligen ein nicht leicht zu lösendes Problem, doch für FH-Absolvierende ein besonderes, da sie nicht in das Netzwerk der Nachwuchsförderung der promovierenden Universität eingebunden sind. Die Chancen von Absolventinnen der Beuth Hochschule eine wissenschaftliche Projekt- oder Mitarbeiterinnenstelle zur Promotion an der promovierenden Universität zu bekommen, waren daher eher gering. Als Möglichkeiten zur Lösung des Finanzierungsproblems boten sich die Bewerbung um ein Stipendium und/oder die Suche eines promotionsbegleitenden Jobs an.

Für Absolventinnen der Beuth Hochschule war oftmals das Hypatia Programm die erste Anlaufstelle.

Das Hypatia Promotionsstipendium in Höhe von 852 Euro und dann ab 2009 in Höhe von 950 Euro entsprach einer Grundsicherung. Hypatia Promotionsstipendiatinnen übten deshalb vielfach zusätzlich eine Berufstätigkeit aus, um Krankenkassenbeiträge sowie andere laufende Kosten finanzieren zu können, nur wenigen standen ergänzende private Mittel zur Verfügung.

Im Ergebnis schlossen nur einzelne Hypatia Promotionsstipendiatinnen in der Förderlaufzeit von 2 bzw. $21 / 2$ Jahren, wenn sie noch in den Genuss eines Vorbereitungsstipendiums gekommen waren oder ein 6-monatiges Abschlussstipendium erhielten, ihre Promotion erfolgreich ab.

Der Satz für Promotionsstipendien von Begabtenförderungswerke lag immer über dem des Hypatia Programms. Allerdings wird von den Begabtenförderungswerken die Höhe des Stipendiums einkommensabhängig berechnet, das Hypatia Stipendium war einkommensunabhängig.

Derzeit liegt der Promotionsstipendium-Höchstsatz der Begabtenförderungswerke bei 1.150

${ }^{33}$ Formale Vergabekriterien Hypatia Programm, abgestimmt auf den FFK-Sitzungen 17.12.2008 und 16.02.2009 
Euro. Zusätzlich erhalten die Stipendiaten und Stipendiatinnen eine Forschungskostenpauschale in Höhe von 100 Euro und ggf. werden ein Familienzuschlag von 155 Euro und/oder Kinderbetreuungskosten ab 155 Euro gezahlt. Die Höchstförderungsdauer sind vier Jahre (2 Jahre Grundförderung plus 2 × 6 Monate Weiterförderung aus fachlichen Gründen und ein Jahr aufgrund von Kinderbetreuung oder Behinderung).

Die Zahl der Beuth-Absolvierenden, die ein Promotionsstipendium eines Begabtenförderungswerkes erhielten, müsste zusätzlich zu dieser Studie recherchiert werden.

Ein Erstgutachter bzw. eine Erstgutachterinnen ist gefunden, die Zulassung der promovierenden Universität liegt vor, die Finanzierung ist gesichert, alle Zulassungsauflagen wurden erfolgreich erfüllt, der eigentlichen Arbeit an dem Promotionsthema steht damit nichts im Wege. Die Höhe und Tiefen im Verlauf eines qualifizierenden Forschungsvorhabens zur Promotion erfahren sicherlich nicht nur promovierende mit einem FH-Abschluss. Die umfassende, intensive wissenschaftliche Bearbeitung eines Forschungsthemas ist eine Herausforderung, die es zu meistern gilt.

Besonders wichtig für die Hypatia Promotionsstipendiatinnen blieb auch in dieser Promotionsphase die Betreuung des Professors bzw. der Professorin der Beuth Hochschule, den bzw. die sich die Stipendiatinnen ausgewählt hatten. Im günstigsten Fall übernahmen Beuth Mentoren oder Beuth Mentorinnen Zweit- oder Drittgutachterinnen und Gutachter Aufgaben im Promotionsverfahren ihrer Mentees.

Für die Hypatia Stipendiatinnen gewährleistete des Weiteren das überfachliche Begleitprogramm zur Hypatia Förderung eine institutionelle Anbindung an die ihnen vertraute Hochschule. Zum Hypatia Begleitprogramm gehörten Coaching- und Betreuungsangebote sowie fachliche und überfachliche Seminar- und Workshop-Angebote. Trotz kontinuierlicher Nachfrage durch die promovierenden Beuth-Absolventinnen musste das Begleitprogramm aufgrund fehlender Mittel Ende 2009 - Mittel aus dem quotierten Bereich des BCP wurden hierfür ab 2010 nicht mehr bewilligt - eingestellt werden. 
Auf die absehbaren Veränderungen der BCP Bewilligungspolitik zur Bewilligung von Stipendien reagierte das GuTZ 2014 mit der Initiative zur Institutionalisierung eines Promotionskollegs an der Beuth Hochschule. In Kooperation mit der Leiterin der Forschungsabteilung und eines Professors aus dem FB VIII erarbeitete das GuTZ nach Rücksprache mit dem Präsidium einen Satzungsentwurf für ein Promotionskolleg. In die Formulierung der Präambel und der Ziele für das Kolleg flossen die langjährigen Erfahrungen der Hypatia Promotionsförderung ein.

In der Präambel des Satzungsentwurfs für das angedachte Promotionskolleg wurde ausgeführt:

„Die Beuth Hochschule für Technik Berlin (Beuth Hochschule) richtet ein Promotionskolleg "Leben in der Stadt der Zukunft" ein. Mit dem Promotionskolleg (Kolleg) schafft die Beuth Hochschule einen strukturierten Forschungsraum. Übergeordnetes Ziel ist es, promovierende Absolventinnen und Absolventen der Beuth Hochschule bei der Durchführung ihrer Promotionsvorhaben zu begleiten und zu unterstützen, Innovationen in Forschung und Lehre zu befördern sowie das Forschungsprofil und die Attraktivität der Beuth Hochschule nachhaltig zu stärken.“34

Ein wichtiger Diskussionspunkt zur Zielformulierung war die Einbindung von Promovierenden in die Lehre der Beuth Hochschule. Eine Vermittlung von Erfahrungen als Lehrende sollte für die Promovierenden eine Berufskarriere in der Lehre und Forschung an einer Hochschule zu einer attraktiven Option werden lassen. Hintergrund für Hervorhebung der Bedeutung einer solchen Maßnahme waren die statistischen Auswertungen zum Verbleib der Hypatia Promotionsstipendiatinnen. Danach wählten die erfolgreichen Hypatia Promotionsstipendiatinnen eher nicht „Wissenschaft als Beruf“, sondern entschieden sich für Berufswege außerhalb der Hochschule.

Mit dem Projekt „Vorbereitung einer Hochschulkarriere für FH-Absolventinnen“, das 2016 angelaufen ist, hat das GuTZ ein weiteres Mal auf die „Abwanderung“von herausragenden Beuth Absolventinnen in nichtwissenschaftliche Tätigkeitsfelder reagiert. Den zu erwartenden Forschungsergebnissen zur „Entwicklung und Umsetzung eines Förder- und Qualifizierungsprogramms für Fachhochschul-Absolventinnen - auch mit Migrationshintergrund - zur langfristigen Gewinnung potentieller Bewerberinnen für Fachhochschul-Professuren“35 kann und soll an dieser Stelle nicht vorgegriffen werden.

Bezugnehmend auf die vorstehende analysierende Betrachtung der Förderverläufe von Hypatia Promovendinnen, Gastdozentinnen und Gastprofessorinnen, drängt sich eine weitere in die Zukunft gerichtete Empfehlung auf: Die bisherigen für alle Studiengänge einheitlichen Förderkonzepte für FH-Absolventinnen sollten überarbeitet werden mit dem Ziel, die fachkulturspezifischen Rahmenbedingungen einer Hochschulschulkarriere aufzunehmen. Dies zeigen die Förderverläufe insbesondere von Hypatia Stipendiatinnen und Hypatia Gastdozentinnen der Architektur und der Biotechnologie. Ein Lösungsansatz wäre die Ausarbeitung eines RahmenFörderkonzepts als Dach für differenzierte Förderkonzepte, die fachspezifische Qualifikationsund Karrierewege konstruktiv aufgreifen und weiterentwickeln.

\footnotetext{
${ }^{34}$ Entwurf Promotionskolleg in der Fassung vom 17.06.2014

${ }^{35}$ vgl. Teil I, A3.1, S. 21
} 


\section{Literatur}

AuBeFuehr „Gendergerechte Personalauswahl und -beförderung. Handreichung für EntscheidungsträgerInnen in Wirtschaft und Wissenschaft“, https://www.diversity.tum.de/

fileadmin/w00blt/www/Karriere_und_Weiterbildung/Gendergerechte_Personalauswahl_und_Befoerderung_AuBeFuehr.pdf

Beaufaÿs, Sandra (2004). Wissenschaftler und ihre alltägliche Praxis: Ein Einblick in die Geschlechterordnung des wissenschaftlichen Feldes [22 Absätze]. Forum Qualitative Sozialforschung / Forum: Qualitative Social Research, Volume 5 No. 2,

Zitat Abs. 10, http://nbn-resolving.de/urn:nbn:de:0114-fqs0402109. Beaufaÿs, Sandra (2003): Wie werden Wissenschaftler gemacht? Beobachtungen zur wechselseitigen Konstitution von Geschlecht und Wissenschaft

Zitat Abs. 14, http://www.qualitative-research.net/index.php/fqs/article/view/613/1327\#g3

BUKOF: Empfehlung der Bundeskonferenz der Frauen- und Gleichstellungsbeauftragten an Hochschulen (BUKOF) zur geschlechtergerechten Gestaltung von Karrierewegen an Fachhochschulen 24.10.2016, http://www.bukof.de/tl_files/Veroeffentl/16-10-24-Geschlechtergerechte\%20Karrierewege\%20Fachhochschulen.pdfBurkhardt, Anke; Nickel, Sigrun; Berndt, Sarah; Püttmann, Vitus; Rathmann, Annika: Die Juniorprofessur - vergleichende Analyse neuer und traditioneller Karrierewege im deutschen Wissenschaftssystem. Beiträge zur Hochschulforschung. 1-2/2016

CHE Centrum für Hochschulentwicklung: Fachkulturen und weibliche Wissenschaftskarrieren: Erfolgsfaktoren von Frauenförderung sichtbar gemacht. http://www.che.de/cms/?

getObject=260\&strAction=show\&PK_Projekt=1446\&getLang=de [aufgerufen 10.04.2017]

FFK 2004, (Hrsg.): 10 Jahre Hypatia Programm - Förderung von Nachwuchswissenschaftlerinnen an der Technischen Fachhochschule Berlin, Evaluation von 1994 bis 2003, Berlin März 2004

Grote, Birgit: Personalberatung JR Cape: Professorinnen gesucht!? Ideen zur Verbesserung des Berufungsmanagements an Hochschulen. im Rahmen der Jahrestagung der FH-Frauenbeauftragten 2016 in Brandenburg, http://www.jr-cape-group.com/downloads/

birgit-grote---berufungsmanagement-an-hochschu.pdf

GWK 2015, Chancengleichheit in Wissenschaft und Forschung. 20. Fortschreibung des Datenmaterial 2014/2015, S33/92, http://www.gwk-bonn.de/fileadmin/Papers/GWK-Heft-50-Chancengleichheit. pdf

HS-OWL, Personalentwicklungskonzept für promovierte Hochschulmitglieder in der Qualifizierungsphase. Hochschule Ostwestfalen-Lippe. S. 10 https://www.hs-owl.de/fileadmin/aktuelles/rektoratsmitteilungen/Personalentwicklung.pdf [aufgerufen 11.01.2017]

HRK-Umfrage Promotionen von Fachhochschulabsolventinnen und Fachhochschulabsolventen in den Prüfungsjahren 2009, 2010 und 2011. Hrsg. von der Hochschulrektorenkonferenz, Bonn 2013.

HRK-Empfehlung, Grundsätze für ein nachhaltiges Bund-Länder-Programm zur Gewinnung von Professorinnen und Professoren an Hochschulen für angewandte Wissenschaften (HAW) bzw. Fachhochschulen (FH). Empfehlung des 134. Senats der HRK am 13. Oktober 2016, S. 3, https://www.hrk.de/ uploads/tx_szconvention/Empfehlung_FH_Professoren_

Senat_13102016_01.pdf

HRK Hochschulrektorenkonferenz, Hrsg.: Ungewöhnliche Wege zur Promotion? Rahmenbedingungen und Praxis der Promotion von Fachhochschul- und Bachelor-Absolventen, Dez. 2006, Beiträge zur Hochschulpolitik 3/2007

Jeschke, Sabina und Ihsen, Susanne u.a.(Hrsg.): Genderation BeSt. Untersuchung von geschlechterneutralen und gendersensiblen Berufungsstrategien. Projektübersicht und Handreichung zum Verbundvorhaben im Themenschwerpunkt „Frauen an die Spitze“ - Förderbereich des Bundesbildungsministeriums für Bildung und Forschung „Strategien zur Durchsetzung von Chancengleichheit für Frauen in Bildung und Forschung" (o.J.) 
Peus, Claudia; Braun, Susanne; Hentschel, Tanja; Frey, Dieter, Hrsg.: Personalauswahl in der Wissenschaft. Evidenzbasierte Methoden und Impulse für die Praxis, Berlin 2015

StaBu 2016, Statistisches Bundesamt, Bildung und Kultur, Personal an Hochschulen, 2015, erschienen 14.10.2016. Für Fachhochschulen (S. 43) insgesamt 4139 Professorinnen von insgesamt 19.000 Professuren (S. 41). https://www.destatis.de/DE/Publikationen/

Thematisch/BildungForschungKultur/Hochschulen/PersonalHochschulen2110440157004.pdf? blob=publicationFile [aufgerufen 31.01.2017]

De Ridder, Giebisch, Hachmeister, Leichsenring, Ziegele: Fachkulturen und weibliche Wissenschaftskarrieren: Erfolgsfaktoren von Frauenförderung sichtbar gemacht. Centrum für Hochschulentwicklung Che, Vortrag/Präsentation im Rahmen der Tagung „Fachkulturen und weibliche Wissenschaftskarrieren. Bielefeld 25.02.2013.

TUB Technische Universität Berlin: Leistungsbericht 2015, Kapitel 6. Gleichstellung, S. A 14, https:// www.berlin.de/sen/.../berichte.../leistungsberichte/tu_leistungsbericht_2014.pdf

VDMA Berufungsverfahren in den Ingenieurwissenschaften. Ergebnisse einer Tendenzbefragung bei Hochschulleitungen sowie Dekaninnen und Dekanen des Maschinenbaus und der Elektrotechnik. https://www.vdma.org/documents/105628/

12124323 /Berufungsverfahren\%20in\%20den\%20Ingenieurwissenschaften\%20-\%20Ergebnisbericht\%20final.pdf/ae102d5d-5df3-4180-908f-b44faa191db8

Wensierski, Hans-Jürgen von, mit Langfeld, Andreas; Puchert, Lea: Bildungsziel Ingenieurin. Biographien und Studienfachorientierung von Ingenieurstudentinnen. Studien zur Technischen Bildung, Band 2. 420 Seiten. Barbara-Budrich-Verlag. Leverkusen-Opladen. 2015

Wissenschaftsrat: Empfehlung zur Personalgewinnung und -entwicklung an Fachhochschulen, Weimar 21.10.2016, Drs. 5637 - 16, http://www.wissenschaftsrat.de/download/

archiv/5637-16.pdf [aufgerufen 31.01.2017] 


\section{Bisher erschienene Bände der Schriftenreihe des GuTZ}

Jahrgang 2009

Ausgabe 01/2009: Barthel, Karoline: Wer wagt, gewinnt? Geschlechtsspezifische Unterschiede im Entscheidungsverhalten unter Risiko. Dezember 2009

Jahrgang 2010

Ausgabe 02/2010: Pattloch, Annette: „Studieren in der Lounge“ - Wie StudentInnen Ihre Hoch-schule gestalten würden. Eine geschlechterdifferenzierende Exploration.

Dezember 2010

Ausgabe 03/2010: Ehmsen, Stefanie: Die Vielfalt gestalten - Diversity an Hochschulen.

Dezember 2010

\section{Jahrgang 2011}

Ausgabe 04/2011: Gläsener, Katharina: Diverse Teams = Erfolgsteams? Bedingungen für die Interaktion in geschlechts- und nationalitätsgemischten Teams. April 2011

Jahrgang 2012

Ausgabe 05/2012: Ihsen, Susanne; Ducki, Antje: Gender Toolbox. Juli 2012

Jahrgang 2013

Ausgabe 06/2013: Erlemann, Christiane: Studentinnen mit Migrationshintergrund in Chancengleichheitsprojekten. Dezember 2013

Jahrgang 2014

Ausgabe 07/2014:

Buchem, Ilona: Studentische Essays zum Thema Internet und Gesellschaft. Januar 2014

Jahrgang 2017

Ausgabe 08/2017: Brandt, Martina; Ducki, Antje: Gendersensible Gestaltung des Studiengangs „BWL - Digitale Wirtschaft“ an der Beuth Hochschule für Technik Berlin, Juni 2017 


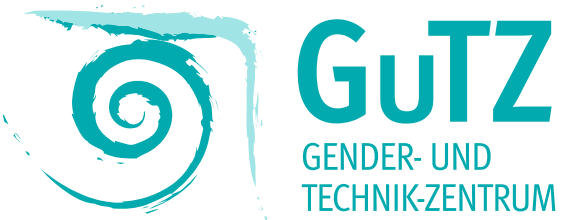

Die Gewinnung von FH-Professorinnen und Professoren wird seit 2016 von Wissenschaftsorganisationen intensiv diskutiert und bundesweit wird nach neuen Ideen und Ansätzen ge-sucht, das sogenannte Nachwuchsproblem zu lösen. Gelingt es an der Beuth Hochschule für Technik Berlin im Rahmen der freien Berufungskorridore den Frauenanteil unter den FH-Professuren markant zu erhöhen und eine „place to be“-Strategie zu etablieren? Aufbauend auf den Erkenntnissen von 22 Jahren Förderung des wissenschaftlichen Nachwuchses im Hypatia Programm wird unter Berücksichtigung von Handlungsempfehlungen zum Personal Recruiting im Wissenschaftsbereich die besondere Situation von Hochschulen der angewandten Wissenschaften im MINT-Bereich berücksichtigt und im Rahmen des Gleichstellungskonzeptes mit Maßnahmen zum Karriereziel FH-Professorin verankert.

\section{Schriftenreihe des Gender- und Technik-Zentrums der Beuth Hochschule für Technik Berlin}

\section{Herausgeberinnen:}

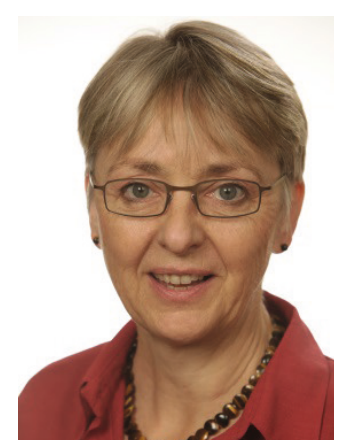

Prof. Dr. Eva-Maria Dombrowski eva-maria.dombrowski@beuth-hochschule.de

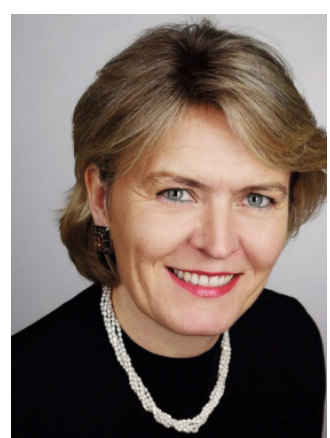

Prof. Dr. Antje Ducki ducki@beuth-hochschule.de

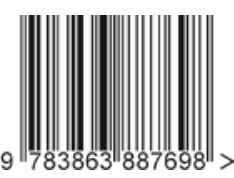

